\title{
Acoustic Optimization for Anti-Phase Asymmetric Rotor
}

\author{
Juntao Xiong 1 \\ Stinger Ghaffarian Technologies, Inc., Moffett Field, CA 94035 \\ Nhan Nguyen ${ }^{2}$ \\ Nicholas B. Cramer ${ }^{3}$ \\ NASA Ames Research Center, Moffett Field, CA 94035
}

\begin{abstract}
This investigation seeks to optimize the implementation of anti-phase alternating trailing edge (TE) patterns for rotor noise suppression. The design objective is to maximize reduction of noise perceived by the community while maintaining the aerodynamic thrust. Computations using a three-dimensional Unsteady-Reynolds-Averaged-Navier-Stokes (URANS) with k- $\omega$ Shear Stress Transport (SST) turbulence model and Ffowcs-Williams and Hawkings (FW-H) formula are used to obtain aerodynamic thrust and far-field noise level. A parametric acoustic study of 13 configurations of KDE rotor with variable alternating trailing edge period, alternating trailing edge length, and trailing edge deflection angle is conducted. The best design candidate for the KDE rotor has a four-period TE waveform which results in a reduction in far-field noise level of $2.1 \mathrm{~dB}$ in the hover condition and a reduction of $1.1 \mathrm{~dB}$ in the forward flight condition at $9.7 \mathrm{~m} / \mathrm{s}$. A further parametric acoustic study is conducted for a different rotor manufactured by APC. Six APC rotor design candidates are simulated. The best design candidate $4 \mathrm{H}$ for the APC rotor results in a reduction in far-field noise level of $4.0 \mathrm{~dB}$ in the hover condition and a reduction of $1.3 \mathrm{~dB}$ in the forward flight condition at $9.7 \mathrm{~m} / \mathrm{s}$. A series of acoustic experiments in the Penn State University (PSU) anechoic chamber have been conducted. In the forward flight condition at $9.7 \mathrm{~m} / \mathrm{s}$, the APC anti-phase $4 \mathrm{H}$ rotor offers clear evidence of noise suppression capability across a wide range of the azimuthal angle. In the broadband frequency range of 2000-4000 $\mathrm{Hz}$, the APC anti-phase $4 \mathrm{H}$ rotor produces as much as $6 \mathrm{~dB}$ noise reduction. The experimental results appear to confirm the noise suppression capability of the proposed anti-phase rotor design concepts.
\end{abstract}

\section{Introduction}

Urban air mobility (UAM) concepts have been proposed as a new mode of transportation to dramatically decrease the door-to-door travel time for short- to mid-distance trips (20 - 200 miles) [1]. The technological development of distributed electric propulsion (DEP) has enabled multi-rotor designs that are capable of reducing the cost of vertical take-off and landing (VTOL) by reducing the complexity of the design, operational cost via fuel savings, and fixed operating cost via insurance reductions [2]. These cost reductions could go a long way to reaching the necessary financial conditions for a commercial market to develop.

Due to market conditions and physical limitations, the Silicon Valley has been proposed as an ideal early market for UAM operations. The analysis has shown that much like helicopters, noise is one of the primary technical challenges that need to be addressed in order to have full public adoption. Antcliff et. al. proposes an acceptable noise margin of $65 \mathrm{dBA}$ at a distance of $250 \mathrm{ft}$ which represents the acceptable noise level of large trucks to the community [3]. This criterion has been adopted by the burgeoning industry [4]. The majority of UAM noise comes from the aircrafts rotors. Rotor noise is a complex problem with many different potential sources though the noise sources are generally grouped into a few categories [5, 6]: loading noise, vortex noise, rotational noise, and blade vortex interaction (BVI).

There have been several approaches to addressing rotor blade noise by altering the design of the rotor blade. Yung has a comprehensive review of different approaches to vortex interaction noise reduction [7]. These include technologies to explicitly disrupt tip vortices like flow spoilers and end plates. There have been various attempts to develop wing tip geometries that would reduce the magnitude to the tip vortices. Investigations into a porous leading edge to create leading edge suction to reduce vortex shedding have been considered. There have also been investigations into the optimization of the whole blade by making the blades bend to further disrupt the tip vortices and the BVI in particular [8]. There have even been proposed solutions for active twist mechanisms to actively reduce the vortex noise [9].

An anti-phase rotor noise reduction concept was proposed by Nguyen to NASA CAS (Convergent Aeronautics Solutions) incubation project in FY 2018 [10]. A preliminary acoustic experiment of an 8-inch rotor was conducted 
A

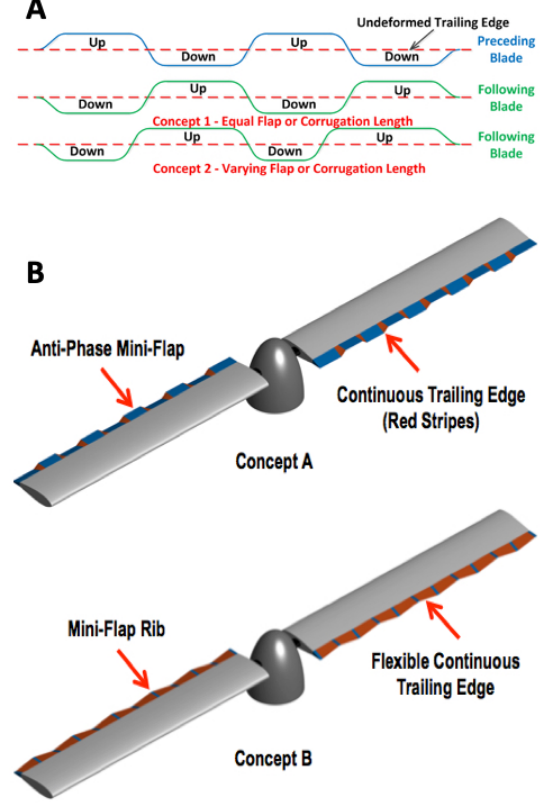

C

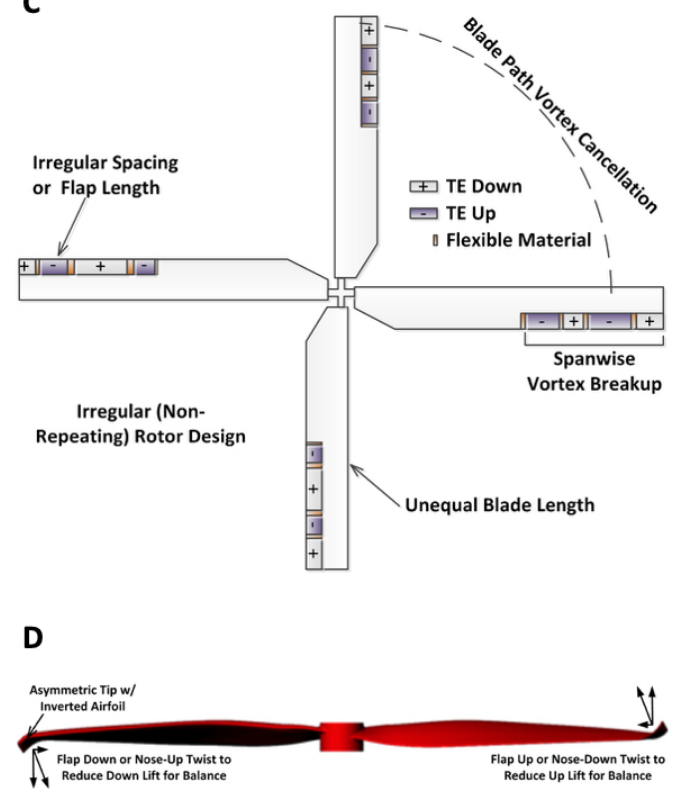

Figure 1 Anti-Phase Rotor Concepts

in 2018 in the LifeSaver open-circuit wind tunnel at NASA Ames Research Center to evaluate the potential merit of this anti-phase rotor concept. Figure 1 illustrates the proposed concept. The anti-phase rotor concept is embodied by a continuous trailing edge (TE) shape modified in an anti-phase alternating pattern from blade to blade so as to prevent harmonic reinforcement of the blade vortex structure that could lead to an acoustic reduction. To verify the noise reduction concept, four different trailing edge configurations are proposed and tested: an asymmetric winglet, and three anti-phase TE shapes which include a corrugated, sine, and adjusted sine waveform pattern, shown in Fig. 2 . The test results indicate that the proposed anti-phase rotor concepts appear to be an effective means of reducing blade vortex interaction noise. The corrugated design appears to be a promising design candidate.

A follow-on effort to further investigate the anti-phase rotor concept is performed in this paper. The investigation has two objectives. The first objective is to conduct computational fluid dynamics (CFD) simulations to investigate the noise characteristics of several anti-phase rotor designs. The goal is to identify the best design candidate that offers the greatest noise reduction among the design candidates evaluated. The second objective is to conduct a series of acoustic tests of one or more anti-phase rotors in an anechoic chamber at Pennsylvania State University (PSU) to evaluate the merit of the anti-phase rotor concept.

\section{Possible Mechanisms of Noise Reduction}

Figure 1 illustrates the proposed anti-phase rotor noise reduction concepts. One anti-phase concept is embodied by a continuous trailing edge shape modified in an anti-phase alternating pattern from blade to blade so as to prevent harmonic reinforcement of the blade vortex structure that could lead to an acoustic reduction. Figure 1 further illustrates two concepts of a two-bladed rotor employing an anti-phase alternating trailing edge pattern. On one blade, the anti-phase trailing edge pattern is $180^{\circ}$ out of phase with respect to the anti-phase trailing edge pattern on the other blade. This is further illustrated in Fig. 1 showing the anti-phase trailing edge patterns of the preceding blade and following blade being out of phase, for example, the trailing edge in a downward deflection on the preceding blade but in an upward deflection on the following blade at the same radial position. In another embodiment, the anti-phase trailing edge pattern does not follow the exact $180^{\circ}$ out-of-phase rule. This is shown by the first line and third line illustrating the anti-phase trailing edge pattern in Fig. 11B. To break up the harmonic reinforcement along the blade span, the anti-phase pattern is applied over several segments of the trailing edge with a constant length or varying length from blade to blade.

The anti-phase trailing edge can be accomplished by either a passive design or active design. A passive design has the advantage of design simplicity and would target a minimum noise impact requirement during hover or descent. The disadvantage of a passive design is the negative impact on the aerodynamic performance of the rotor which could result 


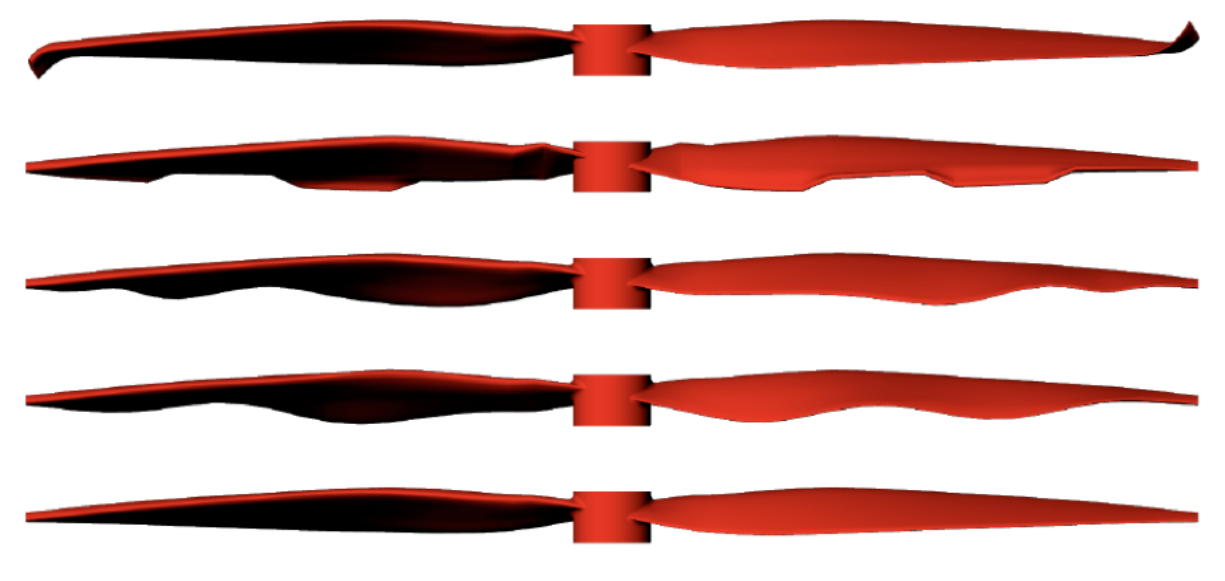

Figure 2 Anti-Phase Rotor Concepts

in increased blade losses, hence increased rotor power. An active design provides more operational flexibility than a passive design. The active design would utilize a series of anti-phase mini-flaps or ribs with a continuous trailing edge feature to provide a mechanism for adjusting the anti-phase trailing edge pattern as needed or restoring the trailing edge to a conventional undeformed state for improved rotor performance during cruise. The continuous trailing edge in an active design could be enabled by a flexible transition section in between the mini-flaps or a flexible skin over the entire trailing edge surface with ribs. Other embodiments are possible. The disadvantage of an active design is the design complexity that could lead to an increase in weight and power for actuation systems. In addition, reliability is another operational factor that could negate some benefits of an active design.

Figure $1 \mathrm{C}$ illustrates a four-bladed rotor that employs anti-phase rotor blades. The anti-phase rotor blades are all dissimilar and can have unequal blade length. This could provide another source for noise reduction.

Because of the nature of the blade loading with the increased tangential velocity toward the rotor blade tip, the tip vortices can be a significant noise source. Swept anhedral tip treatments are effective in reducing acoustic signatures for transonic rotors. The anti-phase rotor concept can be extended to an anti-phase asymmetric blade tip design as shown in Fig. 1D. This concept involves the blade tip of one blade curved in an upward orientation while the blade tip of the other blade curved in a downward position. If the airfoil layout of the rotor blade continues into the curved blade tip regions, a radial force imbalance would result and would cause operational issues. One way to reduce the radial force imbalance due to the asymmetric blade tip design is to employ an inverted airfoil on one of the blade tips. A typical airfoil section with a positive camber is employed everywhere on the main blade surface. This airfoil is transitioned to an airfoil with zero camber near the blade tip region and a negative camber in the blade tip region.

The mechanisms of noise reduction of the anti-phase rotor concepts could be explained by Fig. 3. A two-dimensional (2D) rotor CFD simulation is performed using STAR-CCM+. Two rotor airfoils at a given radial station are modeled using a periodic boundary condition at the inlet of the preceding rotor airfoil with a downward deflected trailing edge and at the exit of the following rotor airfoil with an upward deflected trailing edge, as shown in Figs. $3 \mathrm{~A}$ and $3 \mathrm{~B}$. The velocity contour plot of the CFD simulation is shown in Fig. 3B. It can be clearly seen that anti-phase rotor design creates a non-planar wake that effectively makes the anti-phase rotor design exhibit a similar wake behavior as a stacked co-rotating rotor design, which has recently been proposed by Uber as a means for rotor noise suppression [5]. Figure $3 \mathrm{~B}$ shows a wake with an increased strength shed off the preceding airfoil while the following blade sheds off a much weaker wake. Because of the downward deflected trailing edge of the preceding airfoil, its wake completely bypasses the following blade instead of impinging on the following blade. The two wakes of unequal strength can be seen to be non-interacting, thereby reducing the blade vortex interaction. Figure $3 \mathrm{C}$ illustrates the non-interacting downwash and upwash created by the downward and upward deflected trailing edges of the preceding and following blades, respectively.

Another possible mechanism of noise reduction is the lift creation of a downward-deflected TE in comparison to that of an upward-deflected TE. An airfoil tends to perform more efficiently with a downward-deflected TE up to a certain deflection than with an upward-deflected TE. The $L / D$ ratio tends to increase as the TE is deflected downward to a certain value. A downward-deflected TE has been used as a mechanism for drag reduction for fixed-wing aircraft 


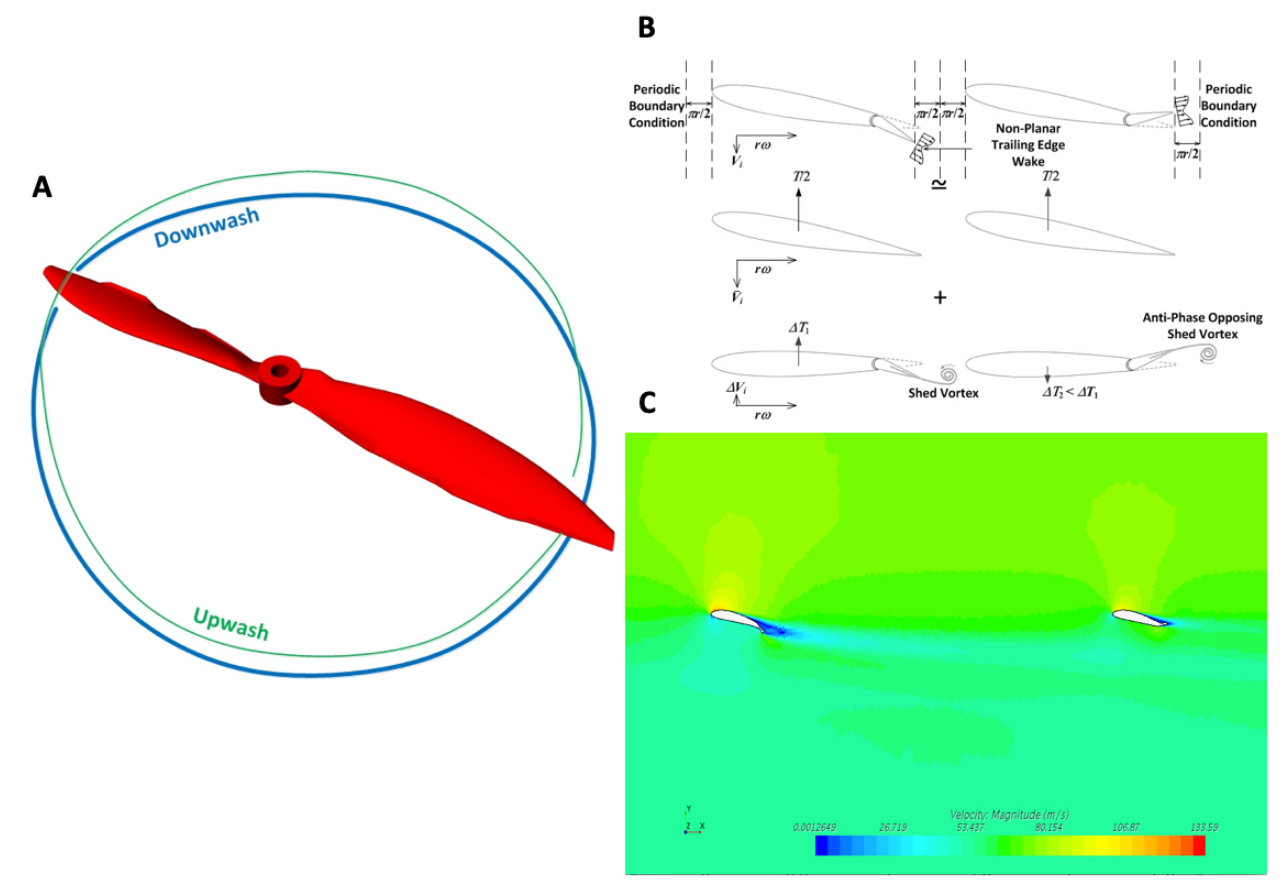

Figure 3 Possible Mechanism of Noise Reduction of Anti-Phase Trailing Edge Rotor Concept

[6]. As the trailing edge deflects upward, the "kink" in the lower surface could induce an early flow separation that effectively reduces the upwash angle of the flow exiting the trailing edge. The net effect is that for the same downward and upward deflection of the trailing edge, the incremental up-lift created by the downward-deflected trailing edge is greater than the incremental down-lift created by the upward-deflected trailing edge. This results in a net overall increase in thrust at the same rotor speed. Conversely, the rotor speed can be reduced to achieve the same amount of thrust, thereby lowering the tonal noise generated by the blade passing frequency.

The mechanism of noise reduction of the asymmetric blade tip design could be explained by Fig. 4 . A high-fidelity CFD simulation of the asymmetric blade tip design is performed using LAVA, a NASA Ames high-fidelity CFD code. The vorticity contour plot of the asymmetric blade tip design is compared to that of the corresponding conventional straight blade design. It may be apparent from Fig. 4 that the vorticity at the blade tip of the conventional straight blade design, shown in Fig. 4A has a higher accumulation than that of the asymmetric blade tip design as shown in Fig. $4 \mathrm{~B}$, which could suggest that the reduction in the harmonic reinforcement of the blade tip vortex structure could be a possible mechanism of noise reduction of the asymmetric blade tip design. Figure $4 \mathrm{C}$ illustrates a pair of co-rotating tip vortices, which create an offset between the two tip vortices. This offset thus effectively mitigates the effect of the following blade flying into the wake created by the tip vortex of the preceding blade, thereby resulting in lower noise.

\section{Numerical Investigation}

To better understand the noise reduction mechanisms of the anti-phase rotor concept and to achieve the maximum noise reduction at a matching thrust requirement, an acoustic optimization study is performed using the commercial CFD software STAR-CCM+. The investigation is performed using a three-dimensional (3D) Unsteady Reynolds-Averaged Navier-Stokes (URANS) commercial solver STAR-CCM+ with $k-\omega$ Shear Stress Transport (SST) turbulence model and Ffowcs-Williams and Hawkings (FW-H) formula to obtain aerodynamic thrust and far-field noise level. Two parametric acoustic studies of two different rotor designs are conducted. The first rotor design is a rotor manufactured by KDE which uses a proprietary geometry. The geometry initial KDE rotor furnished by PSU is shown in Fig. 5 . The CAD geometry of the KDE rotor is created from a laser scanning measurement performed by PSU. The second rotor design is a rotor manufactured by APC who furnishes the rotor geometry. The geometry of the APC rotor, shown in Fig. 6 , is provided by APC through PSU. Both rotors are nominally 18 inches in diameter. 
A

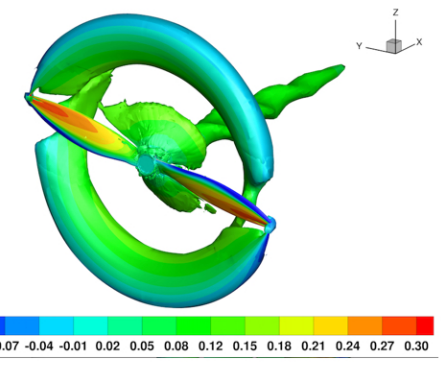

Vorticity of Conventional Blade Computed by LAVA

C

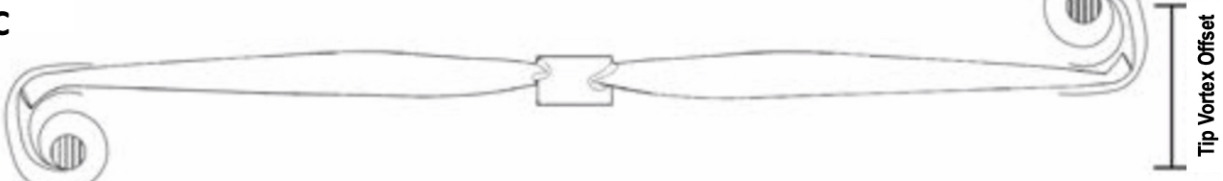

Figure 4 Possible Mechanism of Noise Reduction of Anti-Phase Asymmetric Tip Rotor Concept

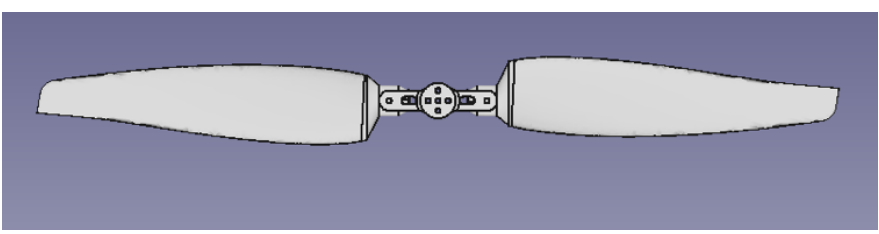

Figure 5 Initial KDE Rotor Furnished by PSU

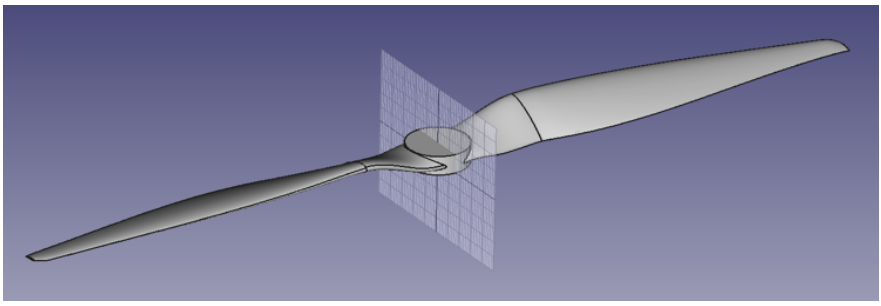

Figure 6 APC Rotor 


\section{A. Computational Approach}

\section{Governing Equations}

Due to the expected rotor tip speed to be no greater than Mach 0.31 , the flow is essentially incompressible. The incompressible URANS equations are expressed as follows:

$$
\begin{gathered}
\sum_{i=1}^{3} \frac{\partial u_{i}}{\partial x_{i}}=0 \\
\rho\left(\frac{\partial u_{i}}{\partial t}+\frac{\partial u_{i} u_{j}}{\partial x_{j}}\right)=-\frac{\partial p}{\partial x_{i}}+\frac{\partial \tau_{i j}}{\partial x_{j}}
\end{gathered}
$$

where the viscous shear stress tensor $\tau_{i j}$ is given by

$$
\begin{gathered}
\tau_{i j}=\tau_{i j}^{L}+\tau_{i j}^{T} \\
\tau_{i j}^{L}=\mu\left(\frac{\partial u_{i}}{\partial x_{j}}+\frac{\partial u_{j}}{\partial x_{i}}\right)-\frac{2}{3} \mu \frac{\partial u_{k}}{\partial x_{k}} \delta_{i j}
\end{gathered}
$$

The closure turbulence model used to evaluate the turbulent shear stress $\tau_{i j}^{T}$ and viscosity $\mu$ is the $k-\omega$ SST turbulence model [11]. The SST turbulence model combines the advantages of the $k-\omega$ and $k-\epsilon$ turbulence models to give superior performance in simulating the wall boundary layer and freestream flow, thus the $k-\omega$ SST model is chosen for all the cases in this study. The equations are

$$
\begin{gathered}
\frac{\partial k}{\partial t}+u_{j} \frac{\partial k}{\partial x_{j}}=P-\beta^{*} \omega k+\frac{\partial}{\partial x_{j}}\left[\left(v+\sigma_{k} v_{T}\right) \frac{\partial k}{\partial x_{j}}\right] \\
\frac{\partial \omega}{\partial t}+u_{j} \frac{\partial \omega}{\partial x_{j}}=\frac{\gamma}{v_{T}} P-\beta \omega^{2}+\frac{\partial}{\partial x_{j}}\left[\left(v+\sigma_{\omega} v_{T}\right) \frac{\partial \omega}{\partial x_{j}}\right]+2\left(1-F_{1}\right) \frac{\sigma_{\omega 2}}{\omega} \frac{\partial k}{\partial x_{j}} \frac{\partial \omega}{\partial x_{j}}
\end{gathered}
$$

where

$$
P=\frac{\tau_{i j}}{\rho} \frac{\partial u_{i}}{\partial x_{j}}
$$

The turbulent eddy viscosity is given by

$$
\begin{gathered}
v_{T}=\frac{a_{1} k}{\max \left(a_{1} \omega, \Omega F_{2}\right)} \\
\mu_{T}=\rho v_{T}
\end{gathered}
$$

The turbulent shear stress is calculated using

$$
\tau_{i j}^{T}=2 \mu_{T}\left(S_{i j}-\frac{1}{3} \frac{\partial u_{k}}{\partial x_{k}}\right)-\frac{2}{3} \rho k \delta_{i j}
$$

$F_{1}$ and $F_{2}$ represent blending functions. The model constants can be calculated by

$$
\phi=F_{1} \phi_{1}+\left(1-F_{1}\right) \phi_{1}
$$




\section{Ffowcs Williams-Hawkings (FW-H) Equation}

The Ffowcs Williams-Hawkings (FW-H) equation is the generalized form of Lighthill's acoustic analogy and is derived primarily from Farassat's Formulation 1A. It is the exact rearrangement of the generalized derivatives of the continuity and momentum equations into the form of the inhomogeneous wave equation. The general form of the FW-H equation is [12],

$$
\square^{2} p^{\prime}=\frac{\partial}{\partial t}\left[\rho_{0} v_{n} \delta(f)\right]-\frac{\partial}{\partial x_{i}}\left[l_{i} \delta(f)\right]+\frac{\partial^{2}}{\partial x_{i} \partial x_{j}}\left[T_{i j} H(f)\right]
$$

where $\square^{2}=1 /\left(c_{0}^{2}\right) \partial^{2} / \partial t^{2}-\partial^{2} / \partial x_{k}^{2}$ is the wave operator. $H(f)$ is the Heaviside function. Using the free-space Green's function to compute the sound pressure at the observer's location $x$, the solution can be formulated as

$$
p^{\prime}(\vec{x}, t)=p_{T}^{\prime}(\vec{x}, t)+p_{L}^{\prime}(\vec{x}, t)+p_{Q}^{\prime}(\vec{x}, t)
$$

The first term on the right hand side refers to the monopole (or thickness) term. It arises from the displacement of the fluid as the body passes through. The second term on the right hand side is the dipole (or loading term). It occurs from the unsteady motion of the force distribution on the body surface. Both of these noise sources are surface-related. The third term on the right hand side is the quadrupole (volume source) term and results from the non-linearities present in the flow.

There are two types of surfaces that can be used in the formulation. An impermeable FW-H surface acts as a filter of wall boundary conditions from which the noise originates. This type will produce sound levels only from the monopole and dipole sources. A permeable FW-H surface acts as a filter of internal interface boundaries. This surface is a fine mesh region that surrounds all the sources of noise: monopole, dipole, and quadrupole. In STAR-CCM+, FW-H model uses a concept developed by Brentner and Farassat referred to as the advanced time approach or the source-time-dominant approach. The algorithm looks forward in time to see when the receiver perceives the generated sound waves. This advanced time algorithm makes a distinction between the emission and reception times. The emission times of the acoustic signal from each surface will be constant while the reception time for those signals will be different. As a result, the emission times are fixed and the signals arriving to the receiver are accumulated at their respective emission time slot. The overall acoustic signal at the receiver is the summation of the individual acoustic signal from each of six source surfaces during the same emission time. The goal of FW-H model is to predict the small amplitude acoustic pressure fluctuations at the location of the desired receiver. It predicts the propagation of sound in free space using analytical integral solutions to the generalized wave equation and does not include any effects such as reflections and refractions. This approach is used in the simulations performed in this study to predict noise levels of the receivers in the far-field.

In this study, the governing equations are solved by the commercial CFD solver STAR-CCM+. The finite-difference schemes are second-order accurate in both space and time. The time discretization is second-order implicit backward differencing. The second-order Roe scheme is used for the spatial discretization.

\section{B. Computational Model and Mesh}

Figure 7 shows the computational mesh around the rotor. A cylindrical shape is used to create the extents of the domain. The domain is divided into three regions: the outer domain, the rotating domain, and the permeable domain. The outer domain specifies the volume of air in which the rotor rotates. This volume should be large enough in order for the boundary conditions set to be satisfied at the boundaries. The permeable domain is created for acoustical purposes. In order to include the quadrupole sources in the prediction of the noise levels, a permeable surface needs to be created. This surface's diameter is set to be three times the rotor diameter. The mesh in this domain can be dense enough to avoid acoustic dissipation, while maintaining a relatively coarser mesh in the outer domains. This reduces the number of cells of the mesh and eventually reduces the computational cost of the simulation. The rotating domain is used to specify the rotational speed of the rotor. It is set away from the rotor in order to ensure that there are no changes in the parameters across the interface. The permeable surface would rotate after every time step, causing a re-mesh and change in the computation of fluxes at the interface. The total number of nodes in the 3 domains is about 4 million. A receiver is placed at $1 \mathrm{~m}$ below the rotor center to measure the noise level. Figure 8 shows the receiver location.

The anti-phase rotor geometries are created by deflecting the trailing edges of several spanwise sections of the rotor blades at their three-quarter chord points from the leading edges. A 3D surface loft is then constructed from these rotor blade sections. 


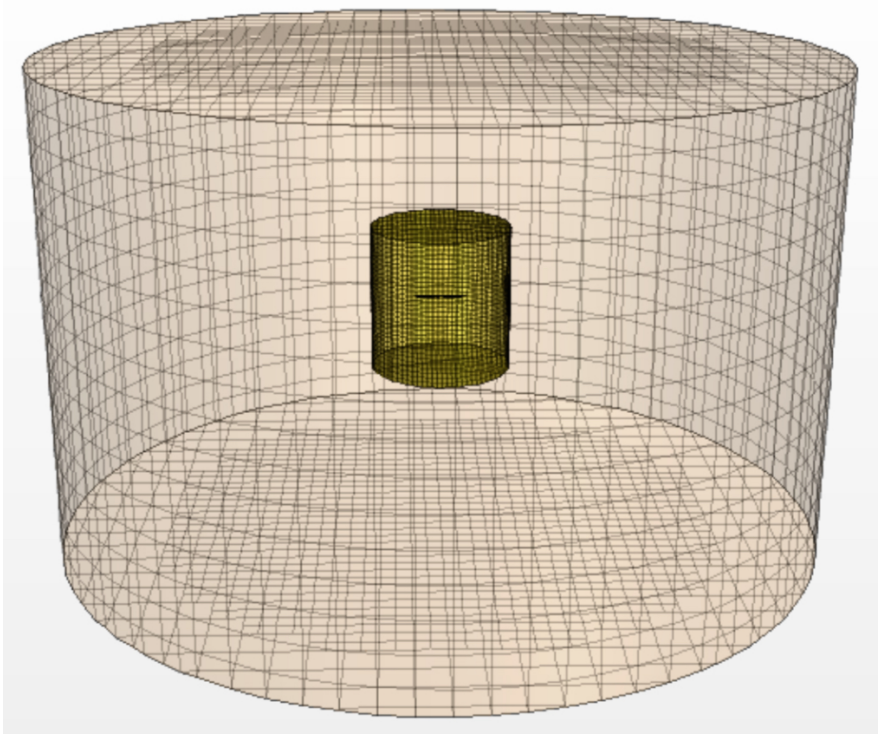

Figure 7 CFD Mesh

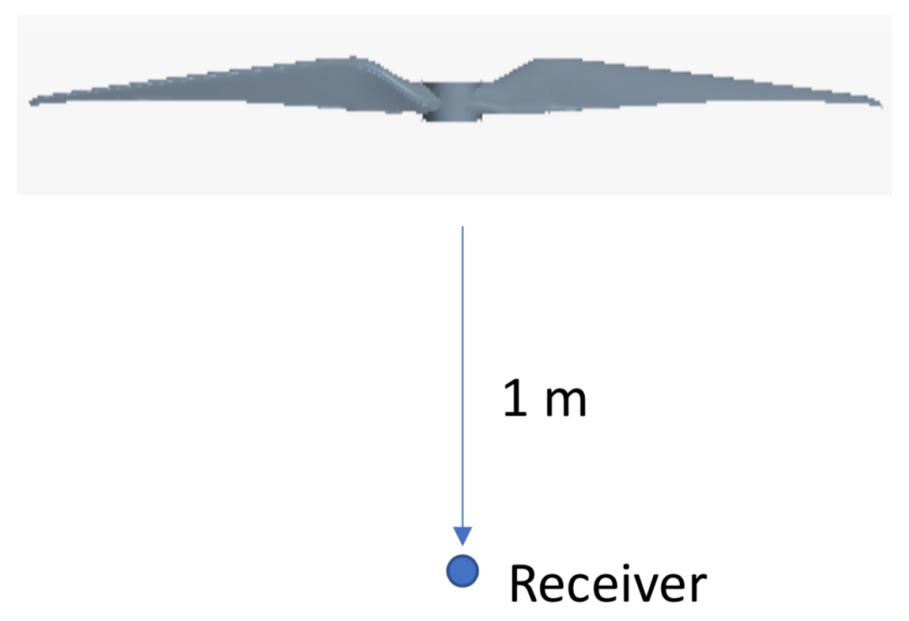

Figure 8 Noise Receiver Location 


\section{Computational Results}

\section{KDE Rotor}

A parametric acoustic study of 13 anti-phase design candidates of the KDE rotor with different alternating TE waveforms, alternating TE length, TE deflection amplitude, and degrees of transition sharpness are listed in Table 1. These design candidates are designated by a numeral and a letter. The numeral designation indicates the number of periods of the TE waveform. Up to five-period TE waveforms are investigated. The letter designation indicates certain physical characteristics of the particular anti-phase TE design. The nominal TE deflection amplitude of the TE waveform is $10^{\circ}$ in the majority of the design candidates. The changes in thrust and noise reduction in hover condition are listed in Table 1 . The rotor speed is 4000 RPM in all cases.

Table 1 shows that as the number of periods in the TE waveform increases up to four while keeping all other parameters the same, the noise reduction increases up to $+2.0 \mathrm{~dB}$ corresponding to design candidate $4 \mathrm{~A}$. The one-period waveform actually results in a noise increase by $1.5 \mathrm{~dB}$. The five-period waveform has a lower noise reduction than the four-period waveform. In all cases, the thrust increases for the design candidates. This is consistent with the hypothesis of the mechanism of thrust increase as discussed previously. The best design candidate in the hover condition is design candidate $4 \mathrm{C}$ which achieves a $5.1 \%$ increase in thrust and $+2.1 \mathrm{~dB}$ noise reduction. Both the thrust increase and noise reduction increase of design candidate $4 \mathrm{C}$ are the largest.

A few design candidates explore the effects of varying the TE deflection amplitude along the blade span, such as: design candidate $3 \mathrm{~B}$, which employs a three-period TE waveform with a varying TE deflection amplitude from $5^{\circ}$ at the blade root to $15^{\circ}$ at the blade tip, design candidate $3 \mathrm{C}$, which employs a reverse TE deflection amplitude, and design candidate $4 \mathrm{C}$, which employs a four-period TE waveform with a varying TE deflection amplitude from $6^{\circ}$ at the blade root to $15^{\circ}$ at the blade tip. It can be seen that, in general, increasing the TE deflection amplitude toward the blade tip produces an increase in the noise reduction. The converse is also true.

The TE deflection amplitude has a strong effect on the noise reduction. As the TE deflection amplitude increases up to a certain value, the noise reduction also increases. This is seen in design candidates 4A and 4B.

All but one design candidate employ equal TE segment lengths for all the TE waveforms. For example, design candidate $4 \mathrm{~A}$ employs a four-period TE waveform with 8 equal TE segment lengths. Design candidate 3D is the only one that explores the effect of varying TE segment lengths which are equal to $0.2 b, 0.167 b$, and $0.135 b$ for the first, second, and third period from the blade root to the blade tip, respectively. The effect of varying TE segment lengths is observed to be minor on the noise reduction.

All but one design candidates employ the standard $180^{\circ}$ phase shift in the TE waveform between the two rotor blades. Design candidate 4D explores the effect of the phase shift and in this case, employs a $90^{\circ}$ phase shift. The decrease in the phase shift from $180^{\circ}$ is seen to produce a decrease in thrust and lower noise reduction. This seems to confirm the merit of the anti-phase rotor design, which implies a $180^{\circ}$ phase shift.

A few design candidates, specifically design candidates $4 \mathrm{E}$ and $4 \mathrm{~F}$, explore the effect of the segment transition characteristics as the TE deflection amplitude changes from a negative to a positive value and vice versa. The transition is indicated by the percentage of the transition length relative to the semi-segment length. Thus, a $0 \%$ transition implies a perfect square wave and a $90 \%$ transition shows a gradual increase (or decrease) in the TE deflection amplitude at the midpoint of the TE segment. All other design candidates employ an $80 \%$ transition. The effect of the transition is observed to produce a small increase in the noise reduction as the transition decreases by comparing design candidates $4 \mathrm{~A}, 4 \mathrm{E}$ and $4 \mathrm{~F}$.

Table 2 shows the computation results for design candidates $4 \mathrm{~A}, 4 \mathrm{E}$, and $4 \mathrm{~F}$ in the forward flight condition at a forward speed of $9.7 \mathrm{~m} / \mathrm{s}$ corresponding to an advanced ratio of 0.1 . The effect of forward flight is seen as an increase in noise and a slight increase in thrust as well for design candidate 4A. In order to compare the noise reduction at the same thrust level, the angle of attack on the rotor is reduced to $-1^{\circ}$. The thrust levels are nearly the same as that of the baseline rotor. All the anti-phase rotor design candidates still show varying levels of noise reduction ranging from $+0.2 \mathrm{~dB}$ for design candidate $4 \mathrm{~F}$ to $+1.1 \mathrm{~dB}$ for design candidate $4 \mathrm{E}$. Based on the results from Tables 1 and 2 , design candidate $4 \mathrm{E}$ appears to offer the best noise reduction in both the hover and forward flight conditions.

Figure 9 shows the TE waveforms of the design candidates of the KDE rotor. Figure 10 shows one instantaneous pressure contour on the blade surface. 


\begin{tabular}{|c|c|c|c|c|c|c|c|}
\hline $\begin{array}{c}\text { Design } \\
\text { Candidate }\end{array}$ & $\begin{array}{c}\text { Number of } \\
\text { Periods }\end{array}$ & $\begin{array}{c}\text { Segment } \\
\text { Length } \\
\text { Fraction } \eta\end{array}$ & $\begin{array}{c}\text { Deflection } \\
\text { Amplitude } \\
\left({ }^{\circ}\right)\end{array}$ & $\begin{array}{c}\text { Phase } \\
\text { Shift } \\
\left({ }^{\circ}\right)\end{array}$ & $\begin{array}{c}\text { Segment } \\
\text { Transition } \\
(\%)\end{array}$ & $\begin{array}{c}\text { Thrust } \\
\text { Change } \\
(\%)\end{array}$ & $\begin{array}{c}\text { Noise } \\
\text { Reduction } \\
(\mathrm{dB})\end{array}$ \\
\hline Baseline & 0 & 1 & 0 & 0 & 0 & 0 & 0 \\
\hline 1A & 1 & $1 / 2$ & 10 & 180 & 40 & +5.0 & -1.5 \\
\hline 2A & 2 & $1 / 4$ & 10 & 180 & 40 & +4.0 & +0.2 \\
\hline 3A & 3 & $1 / 6$ & 10 & 180 & 40 & +2.5 & +0.3 \\
\hline 3B & 3 & $0.175,0.183,0.142$ & $5,10,15$ & 180 & 80 & +4.0 & +0.5 \\
\hline 3C & 3 & $1 / 6$ & $15,10,5$ & 180 & 80 & +5.0 & -0.8 \\
\hline 3D & 3 & $0.2,0.167,0.135$ & $9,12,15$ & 180 & 80 & +2.0 & +0.3 \\
\hline 4A & 4 & $1 / 8$ & 10 & 180 & 80 & +4.0 & +2.0 \\
\hline 4B & 4 & $1 / 8$ & 5 & 180 & 80 & +2.1 & +1.1 \\
\hline 4C & 4 & $1 / 8$ & $6,9,12,15$ & 180 & 80 & +5.1 & +2.1 \\
\hline 4D & 4 & $1 / 8$ & 10 & 90 & 80 & -0.2 & +1.5 \\
\hline 4E & 4 & $1 / 8$ & 10 & 180 & 40 & +3.8 & +2.1 \\
\hline 4F & 4 & $1 / 8$ & 10 & 180 & 90 & +4.1 & +1.9 \\
\hline 5A & 5 & $1 / 10$ & 10 & 180 & 80 & +2.8 & +0.4 \\
\hline
\end{tabular}

Table 1 KDE Anti-Phase Rotor Design Candidates in Hover Condition

\begin{tabular}{|c|c|c|c|}
\hline $\begin{array}{c}\text { Design } \\
\text { Candidate }\end{array}$ & $\begin{array}{c}\text { Angle of } \\
\text { Attack } \\
\left({ }^{\circ}\right)\end{array}$ & $\begin{array}{c}\text { Thrust } \\
\text { Change } \\
(\%)\end{array}$ & $\begin{array}{c}\text { Noise } \\
\text { Reduction } \\
(\mathrm{dB})\end{array}$ \\
\hline 4A & 0 & +4.5 & -2.0 \\
\hline 4A & -1 & -0.1 & +0.6 \\
\hline 4E & -1 & -0.2 & +1.1 \\
\hline 4F & -1 & +0.2 & +0.2 \\
\hline
\end{tabular}

Table 2 KDE Anti-Phase Rotor Design Candidates in Forward Flight Condition at $9.7 \mathrm{~m} / \mathrm{s}$ 


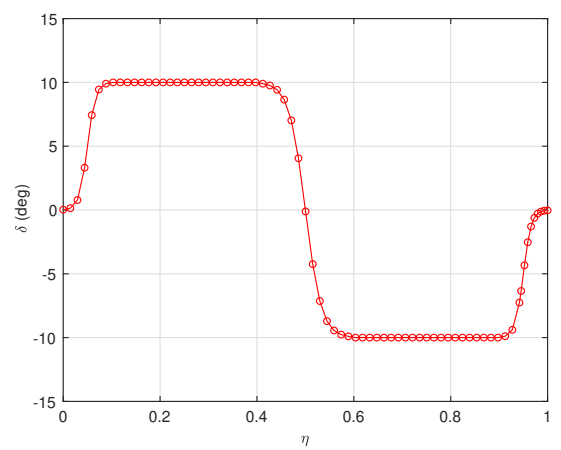

(a) $1 \mathrm{~A}$

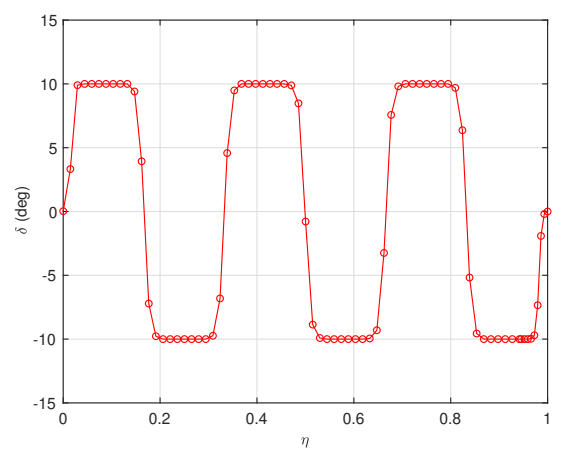

(c) $3 \mathrm{~A}$

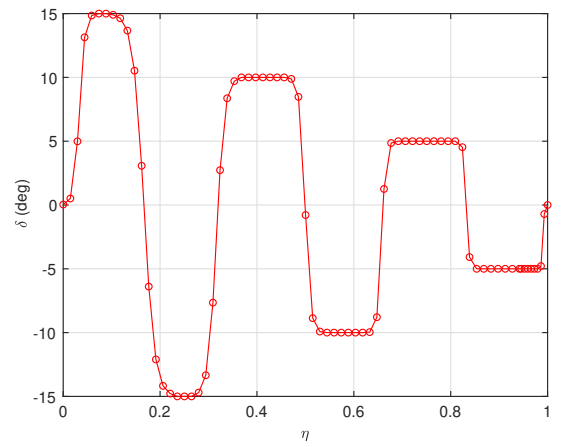

(e) $3 \mathrm{C}$

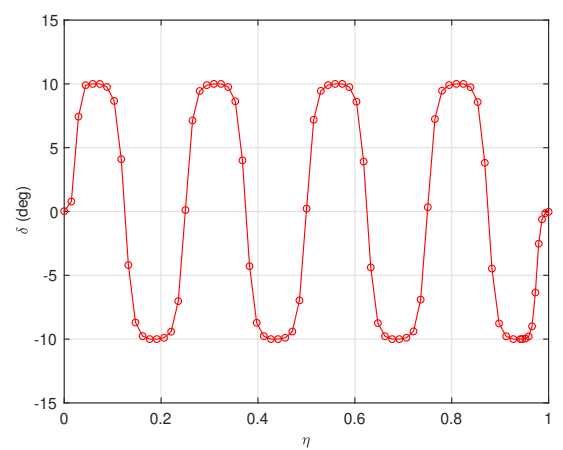

(g) $4 \mathrm{~A}$

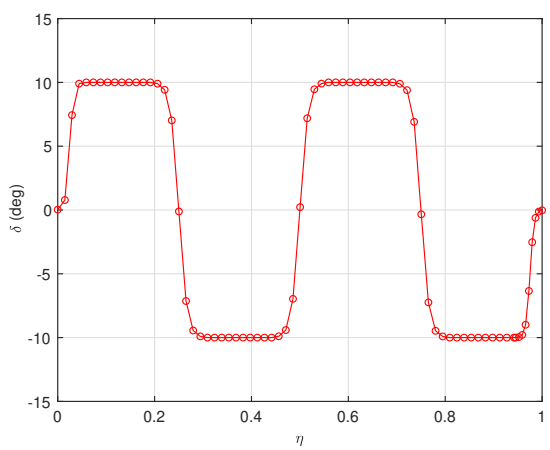

(b) $2 \mathrm{~A}$

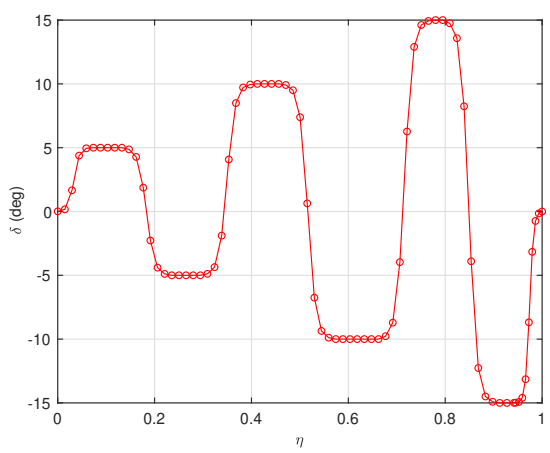

(d) $3 \mathrm{~B}$

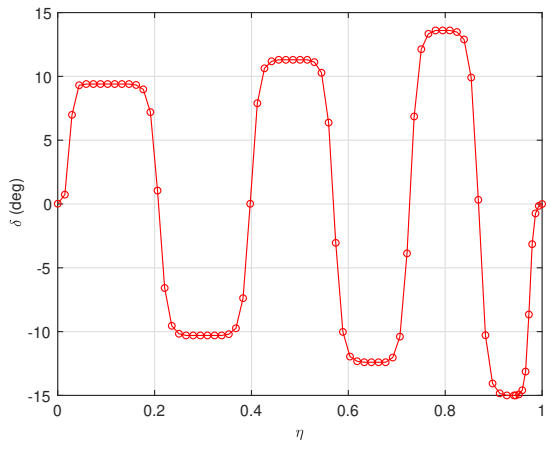

(f) $3 \mathrm{D}$

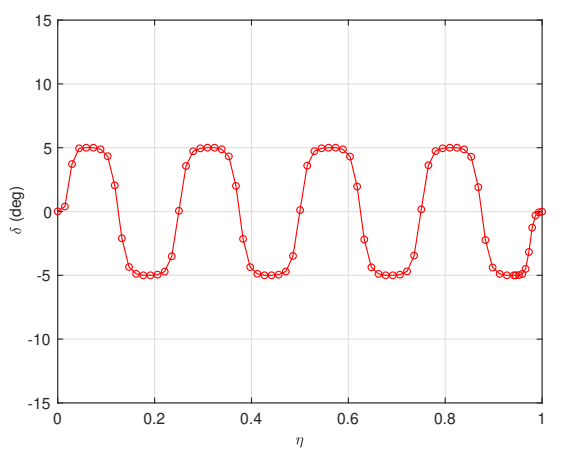

(h) $4 \mathrm{~B}$ 


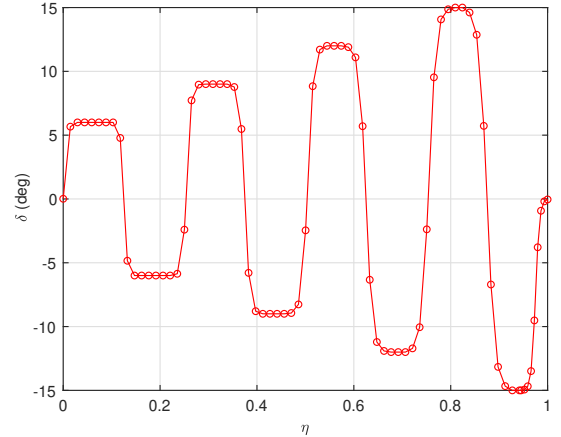

(i) $4 \mathrm{C}$

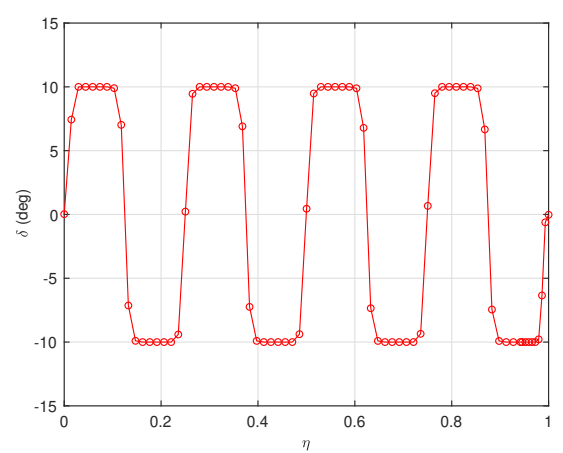

(k) $4 \mathrm{E}$

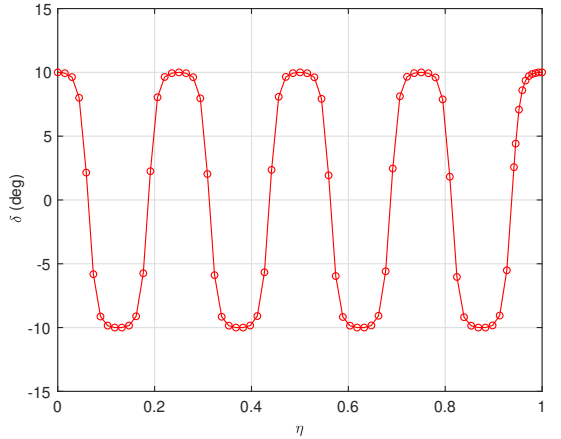

(j) $4 \mathrm{D}$

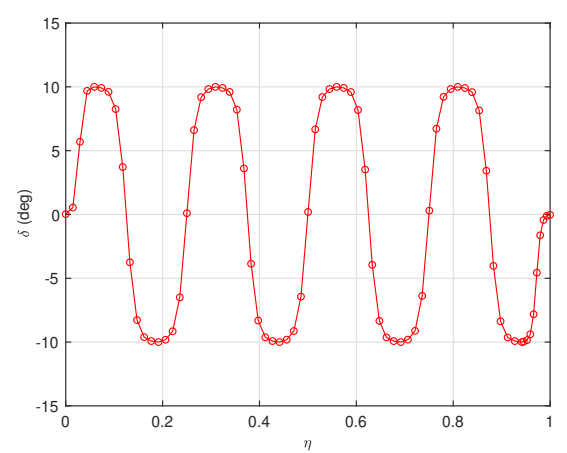

(l) $4 \mathrm{~F}$

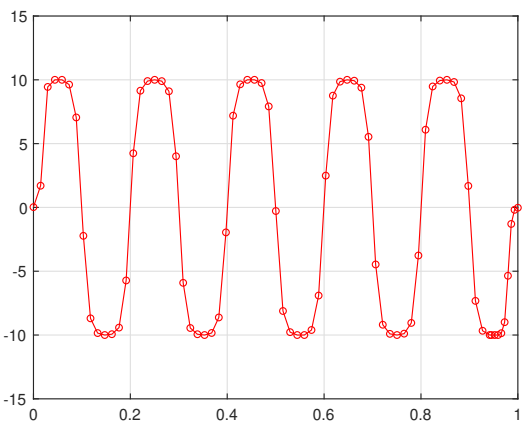

(m) $5 \mathrm{~A}$

Figure 9 KDE Anti-Phase Rotor Trailing Edge Waveforms 


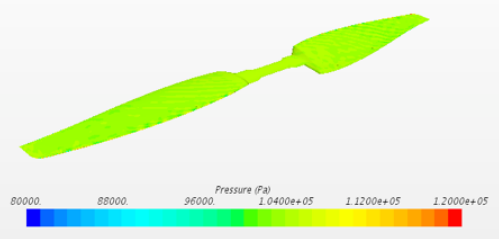

(a) Baseline

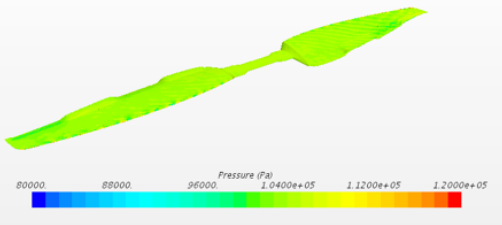

(c) $2 \mathrm{~A}$

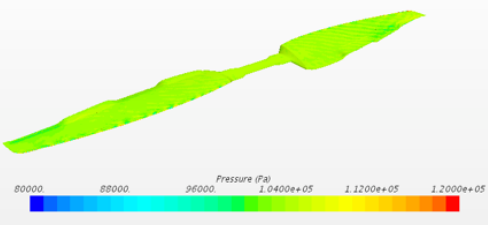

(e) $3 \mathrm{~B}$

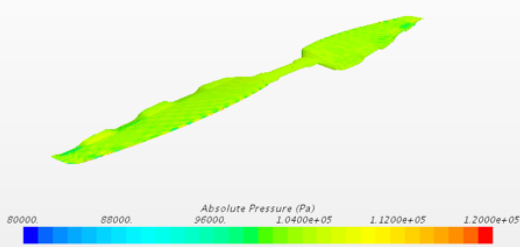

(g) $3 \mathrm{D}$

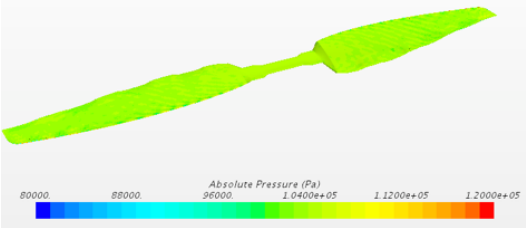

(i) $4 \mathrm{~B}$

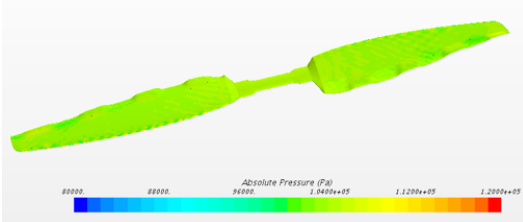

(k) 4D

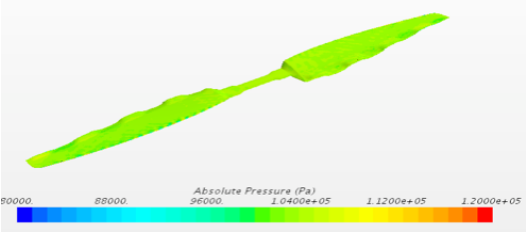

(m) $4 \mathrm{~F}$

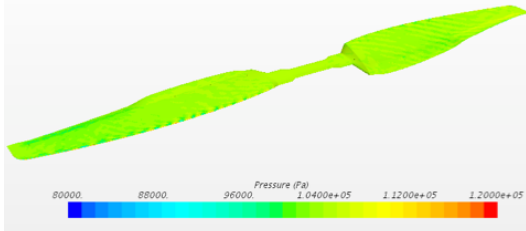

(b) $1 \mathrm{~A}$

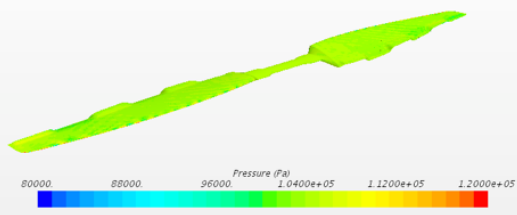

(d) $3 \mathrm{~A}$

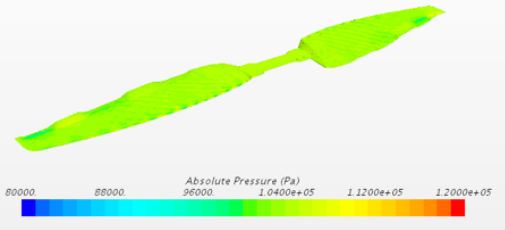

(f) $3 \mathrm{C}$

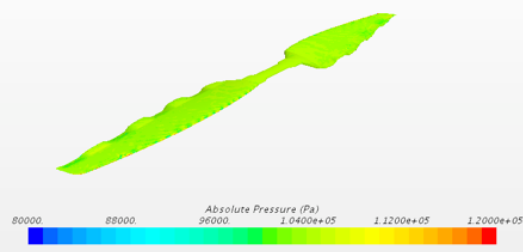

(h) $4 \mathrm{~A}$

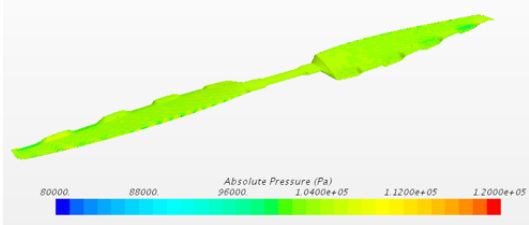

(j) $4 \mathrm{C}$

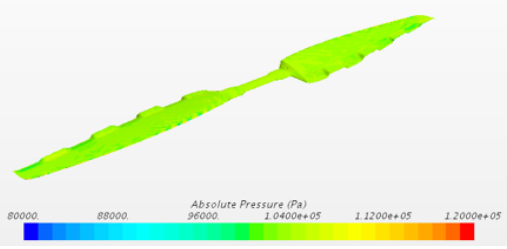

(1) $4 \mathrm{E}$

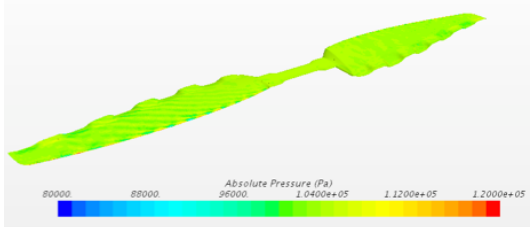

(n) $5 \mathrm{~A}$

Figure 10 Surface Pressure Contours of KDE Anti-Phase Rotors in Hover Condition 
Figure 11 shows the sound pressure level (SPL) spectra of the KDE anti-phase rotors. The noise reduction by the KDE anti-phase rotors can be clearly seen in Fig. 11k k) which compares the SPL of design candidate 4E to the SPL of the baseline rotor. The peak noise amplitude of the baseline rotor at around $5000 \mathrm{~Hz}$ is substantially reduced with design candidate 4E. There is a general decrease in the broadband SPL. The noise reduction by design candidate 4E in the lower frequency range below $2000 \mathrm{~Hz}$ is evident. The performance of design candidate $4 \mathrm{~A}$ is also very similar to design candidate $4 \mathrm{E}$ as shown in $11 \mathrm{~g}$ ). Based on the noise reduction in both the hover and forward flight conditions, design candidate $4 \mathrm{~A}$ is initially selected for the acoustic testing. Later on, design candidate $4 \mathrm{E}$ is investigated and found to perform better than design candidate $4 \mathrm{~A}$ in forward flight conditions. 


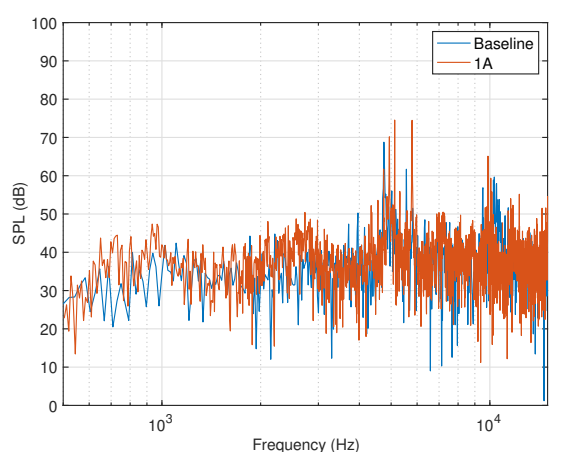

(a) $1 \mathrm{~A}$

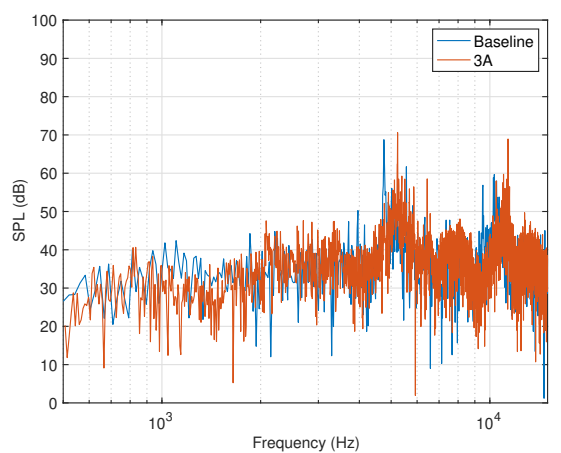

(c) $3 \mathrm{~A}$

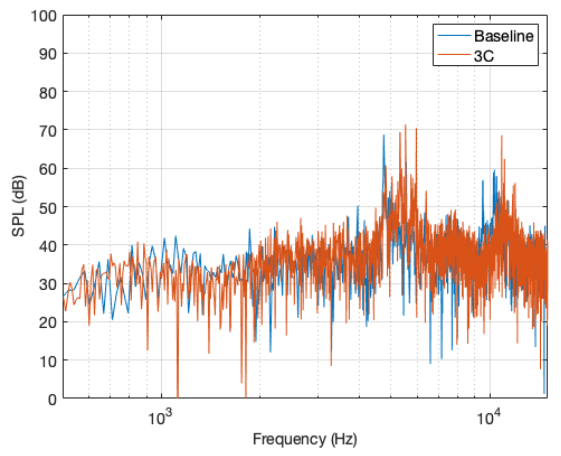

(e) $3 \mathrm{C}$

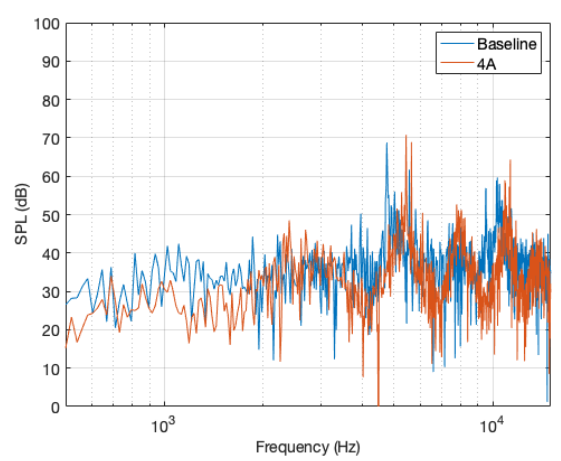

(g) $4 \mathrm{~A}$

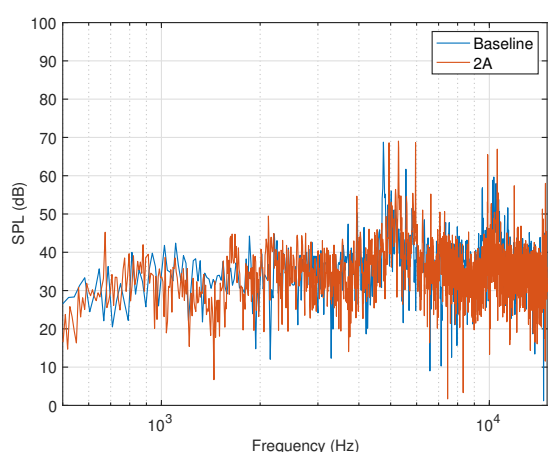

(b) $2 \mathrm{~A}$

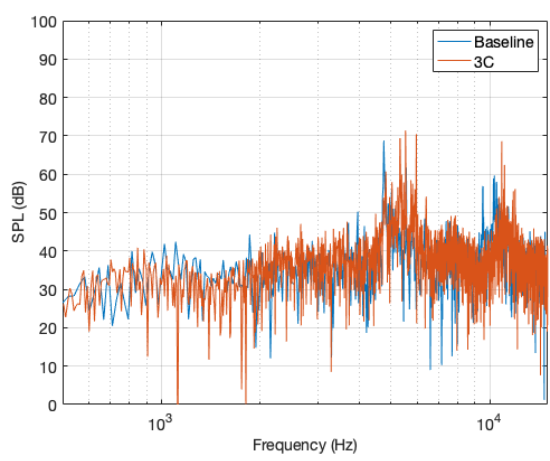

(d) $3 \mathrm{~B}$

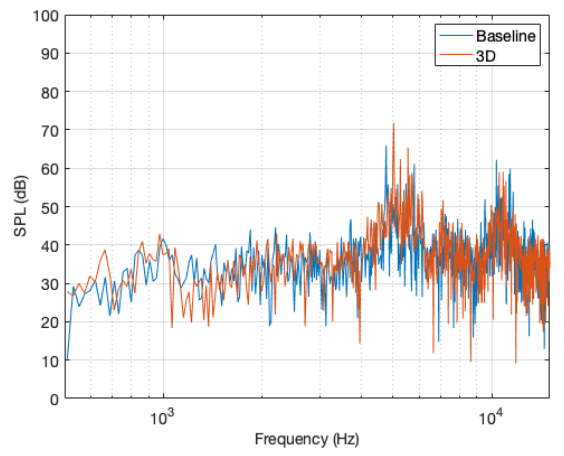

(f) $3 \mathrm{D}$

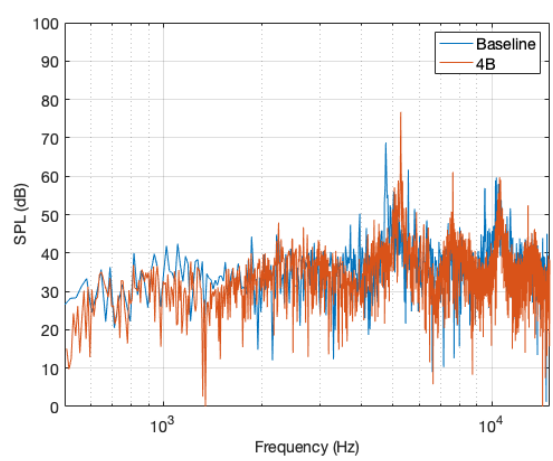

(h) 4B 


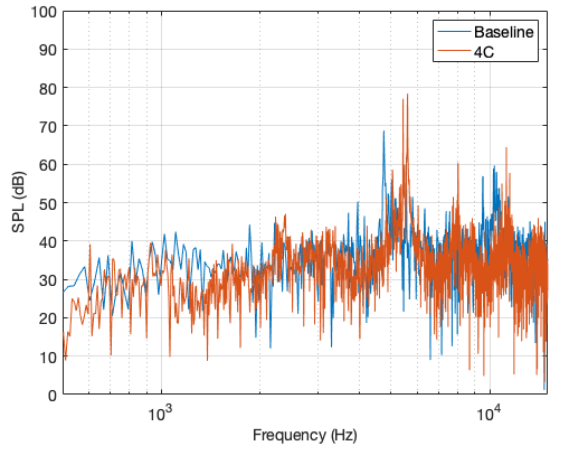

(i) $4 \mathrm{C}$

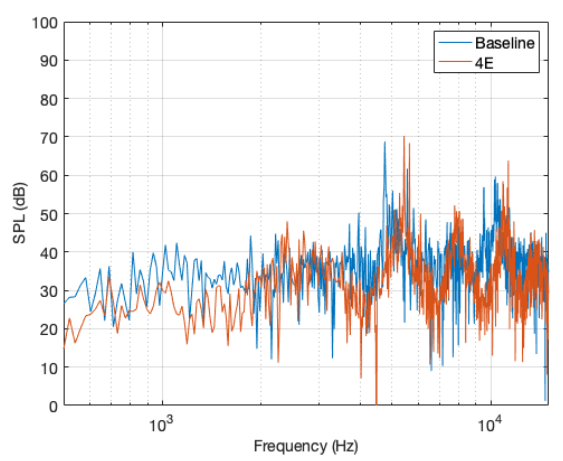

(k) $4 \mathrm{E}$

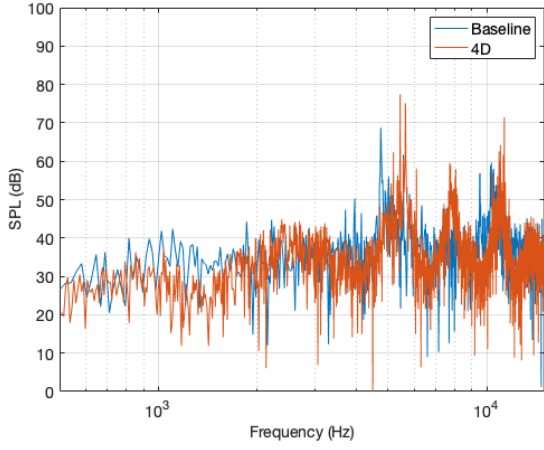

(j) $4 \mathrm{D}$

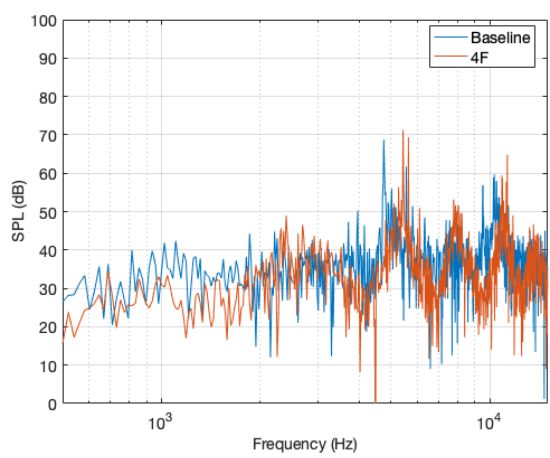

(1) $4 \mathrm{~F}$

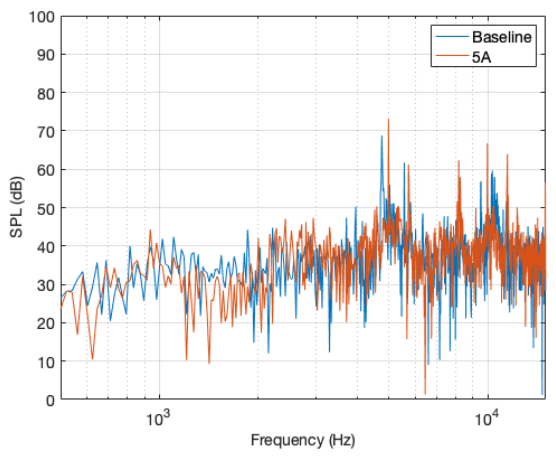

(m) $5 \mathrm{~A}$

Figure 11 Sound Pressure Level Spectra of KDE Anti-Phase Rotors in Hover Condition 


\section{APC Rotor}

A small number of design candidates for the APC rotor are investigated. Using the results for the KDE anti-phase rotors as a guide, the design candidates for the APC rotors are selected to all have a four-period TE waveform, which performs better than other TE waveforms. The APC rotors are simulated at 3200 RPM. The computational results for six different design candidates are shown in Table 3 . Comparing the APC design candidates $2 \mathrm{~A}$ and $4 \mathrm{~A}$ to the corresponding $\mathrm{KDE}$ anti-phase design candidates, the APC design candidates appear to produce slightly more noise reduction that of the corresponding KDE design candidates while the thrust levels between the two sets of design candidates are similar.

Three new design candidates are investigated with the APC rotor: design candidates 4G, 4H and 4I. Design candidate $4 \mathrm{G}$ employs a larger TE deflection amplitude of $15^{\circ}$. At this TE deflection amplitude, it appears that the blade is near or in stall. This is evident in the reduced thrust level from that for design candidate 4E, which has a TE deflection of $10^{\circ}$. The noise reduction is slightly better with design candidate $4 \mathrm{G}$ than $4 \mathrm{E}$, but the blade performance in stall is hardly desirable.

An interesting observation is seen with design candidate $4 \mathrm{H}$. All of the design candidates up to now employ TE waveforms that start at the blade root and end at the blade tip at a TE deflection of zero as shown in Fig. 9. Design candidate $4 \mathrm{H}$, however, does not transition to a TE deflection of zero. Instead, the TE deflection of the last segment at the blade tip is kept constant all the way to the blade tip as shown in Fig. 12) (e). As a result, the positive lift created at one of the blade tips of the anti-phase rotor generates a tip vortex roll-up. At the same time, the negative lift created at the other blade tip generates a tip vortex roll-down. The net effect is that these two vortices are non-coplanar, thereby reducing the blade vortex interaction, similar to the co-rotating tip vortices illustrated in Fig. $4 \mathrm{C}$. Design candidate $4 \mathrm{H}$ achieves a $4.0 \mathrm{~dB}$ noise reduction, the largest of all the design candidates investigated so far. This result coupled with the test data for the anti-phase rotor with asymmetric tips conducted in 2018 [10] strongly suggests that the asymmetric lift creation at the rotor blade tip could be a strong mechanism for noise reduction.

Design candidate 4I employs the in-phase blades with a four-period TE waveform and the last segment at the blade tip deflected downward. This design produces more thrust because the in-phase two blades create positive lift. And this candidate achieves a $2.5 \mathrm{~dB}$ noise reduction.

Table 4 shows the computational results for design candidates $4 \mathrm{H}$ and $4 \mathrm{I}$ in the forward flight condition at a forward speed of $9.7 \mathrm{~m} / \mathrm{s}$. The design candidate $4 \mathrm{H}$ shows $1.3 \mathrm{~dB}$ noise reduction. The design candidate $4 \mathrm{I}$ shows a slight noise increase. Based on the results from Tables 3 and 4 design candidate $4 \mathrm{H}$ appears to offer the best noise reduction in both the hover and forward flight conditions.

Figure 12 shows the TE waveforms of the design candidates of the APC rotor. Figure 13 shows the instantaneous vorticity contours of the design candidates of the KDE rotor. Figure 14 shows the sound pressure level (SPL) spectra of the APC anti-phase rotors. As can be seen, there is a general broadband noise reduction in all the APC anti-phase rotors, especially in the lower frequency range below $2000 \mathrm{~Hz}$. The peak noise amplitude of the baseline rotor at around 4000 $\mathrm{Hz}$ is reduced with all the design candidates. The SPL produced by design candidate $4 \mathrm{H}$ is lower than the other design candidates as seen in Fig. 14(e). 


\begin{tabular}{|c|c|c|c|c|c|c|c|c|}
\hline $\begin{array}{c}\text { Design } \\
\text { Candidate }\end{array}$ & $\begin{array}{c}\text { Number of } \\
\text { Periods }\end{array}$ & $\begin{array}{c}\text { Segment } \\
\text { Length } \\
\text { Fraction } \eta\end{array}$ & $\begin{array}{c}\text { Deflection } \\
\text { Amplitude } \\
\left({ }^{\circ}\right)\end{array}$ & $\begin{array}{c}\text { Tip } \\
\text { Deflection } \\
\left({ }^{\circ}\right)\end{array}$ & $\begin{array}{c}\text { Phase } \\
\text { Shift } \\
\left({ }^{\circ}\right)\end{array}$ & $\begin{array}{c}\text { Segment } \\
\text { Transition } \\
(\%)\end{array}$ & $\begin{array}{c}\text { Thrust } \\
\text { Change } \\
(\%)\end{array}$ & $\begin{array}{c}\text { Noise } \\
\text { Reduction } \\
(\mathrm{dB})\end{array}$ \\
\hline Baseline & 0 & 1 & 0 & 0 & 0 & 0 & 0 & 0 \\
\hline 2A & 2 & $1 / 4$ & 10 & 0 & 180 & 40 & +4.7 & +1.0 \\
\hline 4A & 4 & $1 / 8$ & 10 & 0 & 180 & 80 & +3.3 & +1.8 \\
\hline 4E & 4 & $1 / 8$ & 10 & 0 & 180 & 40 & +3.7 & +2.7 \\
\hline 4G & 4 & $1 / 8$ & 15 & 0 & 180 & 40 & +0.5 & +2.9 \\
\hline $4 \mathrm{H}$ & 4 & $1 / 8$ & 10 & 10 & 180 & 40 & +3.2 & +4.0 \\
\hline 4I & 4 & $1 / 8$ & 10 & 10 & 0 & 40 & +3.9 & +2.5 \\
\hline
\end{tabular}

Table 3 APC Anti-Phase Rotor Design Candidates in Hover Condition

\begin{tabular}{|c|c|c|c|}
\hline $\begin{array}{c}\text { Design } \\
\text { Candidate }\end{array}$ & $\begin{array}{c}\text { Angle of } \\
\text { Attack } \\
\left({ }^{\circ}\right)\end{array}$ & $\begin{array}{c}\text { Thrust } \\
\text { Change } \\
(\%)\end{array}$ & $\begin{array}{c}\text { Noise } \\
\text { Reduction } \\
(\mathrm{dB})\end{array}$ \\
\hline $4 \mathrm{H}$ & 0 & +3.0 & +1.3 \\
\hline $4 \mathrm{I}$ & 0 & +3.9 & -0.3 \\
\hline
\end{tabular}

Table 4 APC Anti-Phase Rotor Design Candidates in Forward Flight Condition at $9.7 \mathrm{~m} / \mathrm{s}$ 


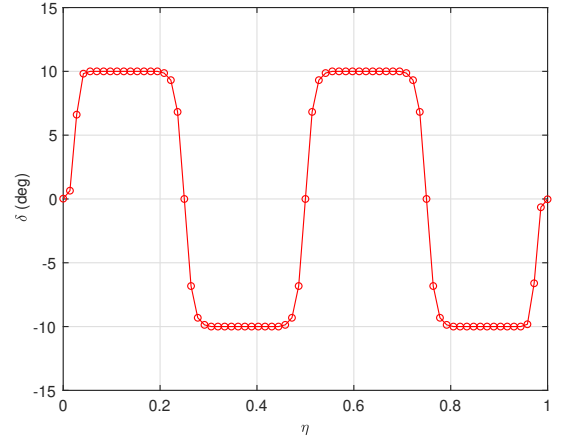

(a) $2 \mathrm{~A}$

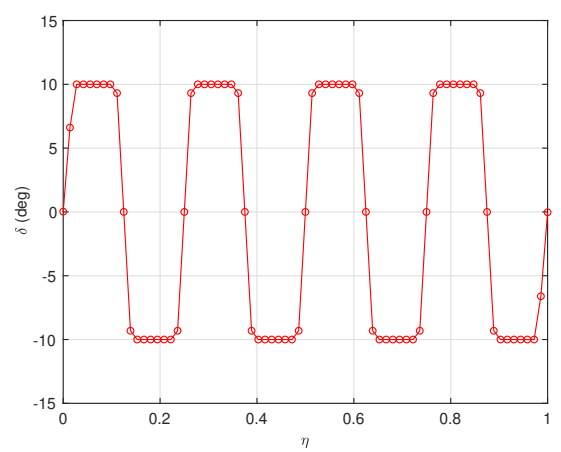

(c) $4 \mathrm{E}$

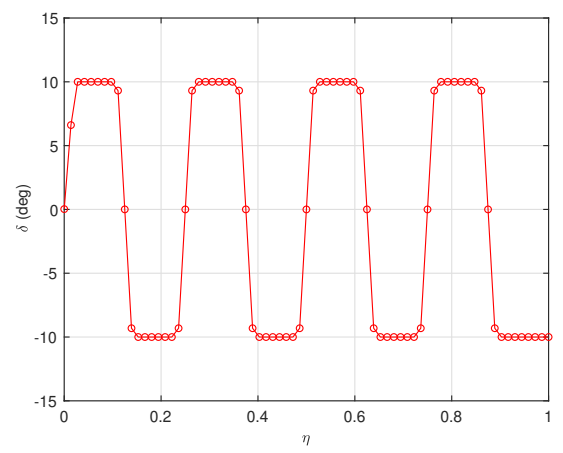

(e) $4 \mathrm{H}$

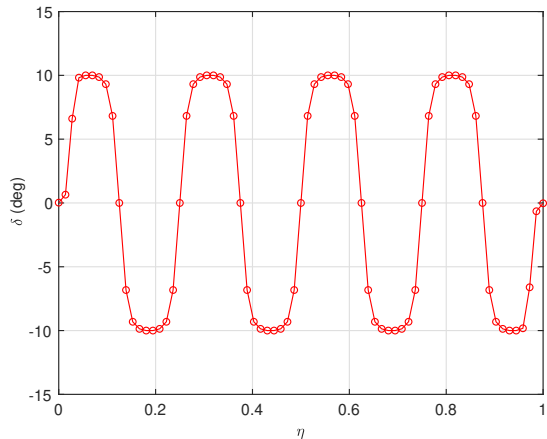

(b) $4 \mathrm{~A}$

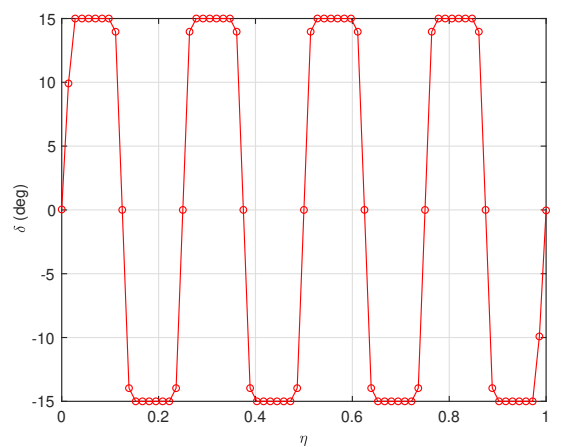

(d) $4 \mathrm{G}$

Figure 12 APC Anti-Phase Rotor Trailing Edge Waveforms 


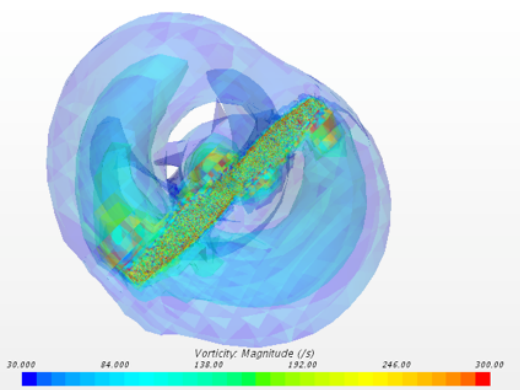

(a) Baseline

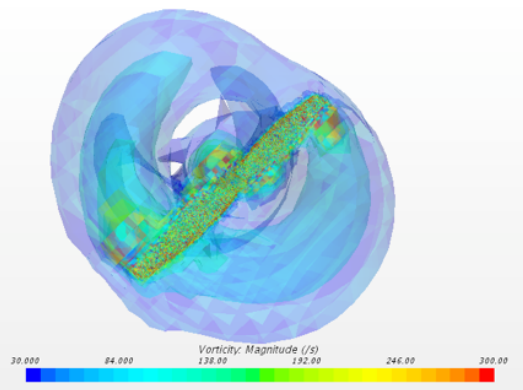

(c) $4 \mathrm{~A}$

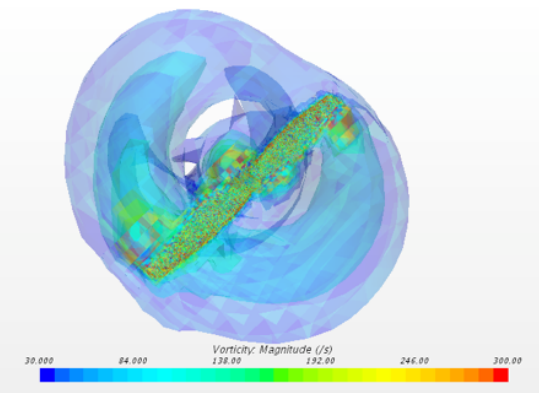

(e) $4 \mathrm{G}$

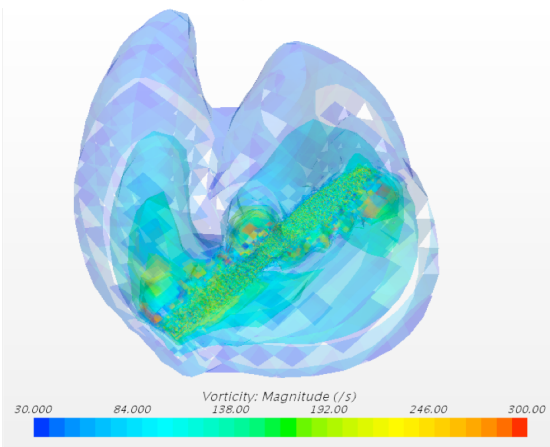

(g) $4 \mathrm{I}$

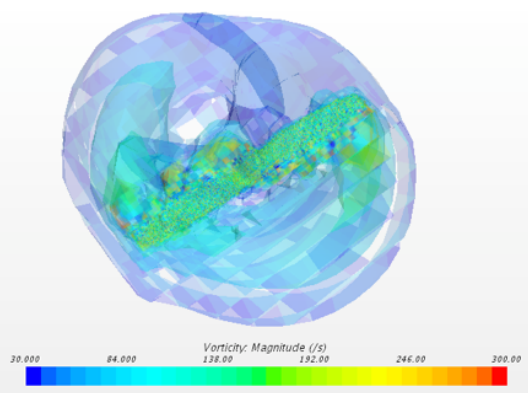

(b) $2 \mathrm{~A}$

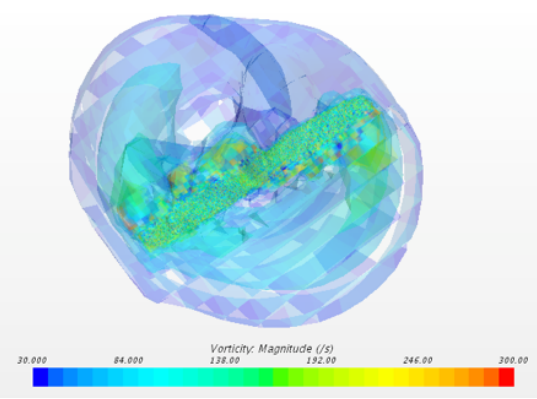

(d) $4 \mathrm{E}$

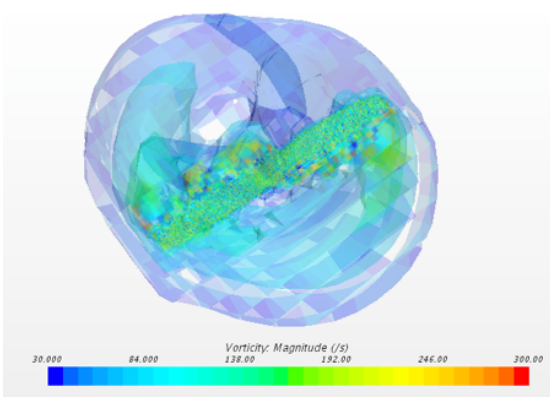

(f) $4 \mathrm{H}$

Figure 13 Instantaneous Vorticity Contours of APC Anti-Phase Rotors 


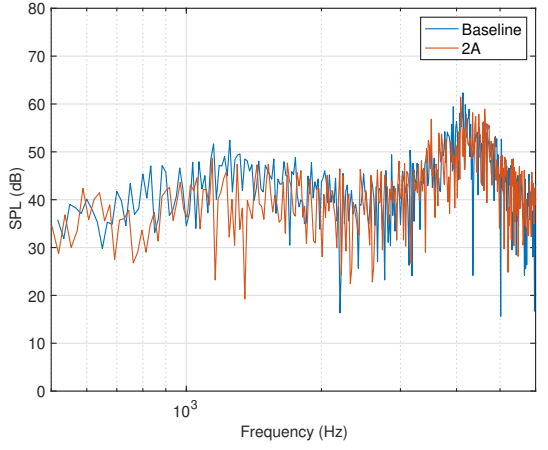

(a) $2 \mathrm{~A}$

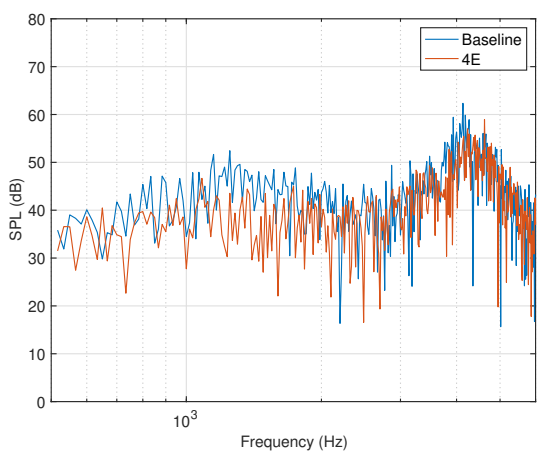

(c) $4 \mathrm{E}$

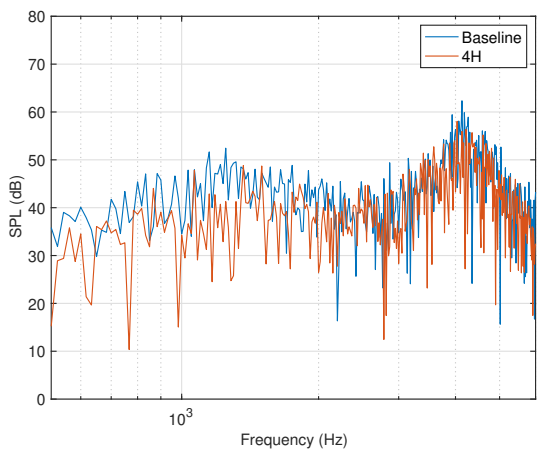

(e) $4 \mathrm{H}$

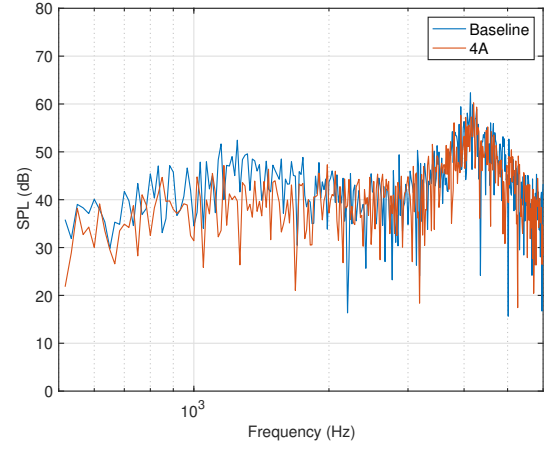

(b) $4 \mathrm{~A}$

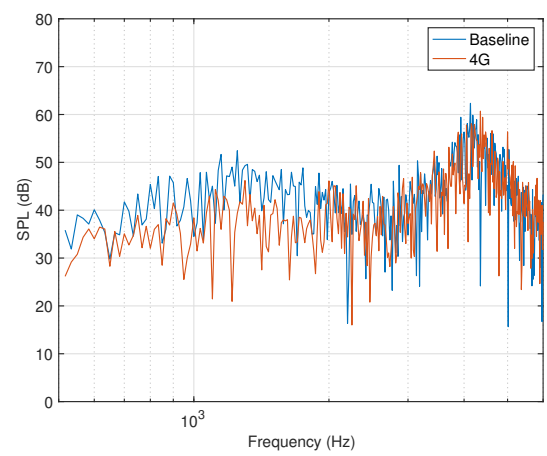

(d) $4 \mathrm{G}$

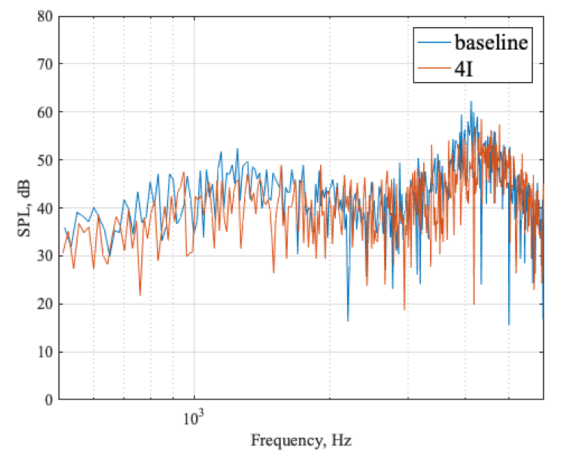

(e) $4 \mathrm{I}$

Figure 14 Sound Pressure Level Spectra of APC Anti-Phase Rotors in Hover Condition 


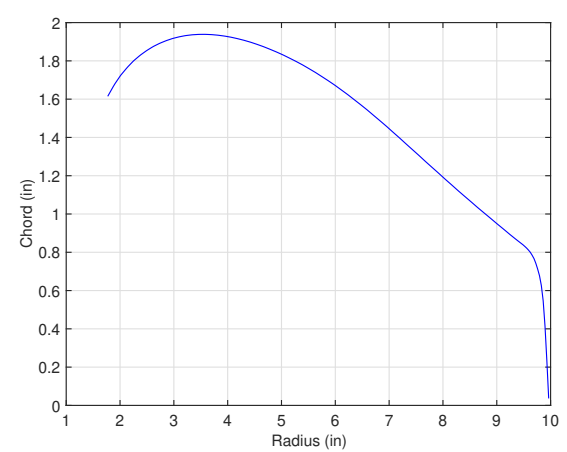

(a) Chord

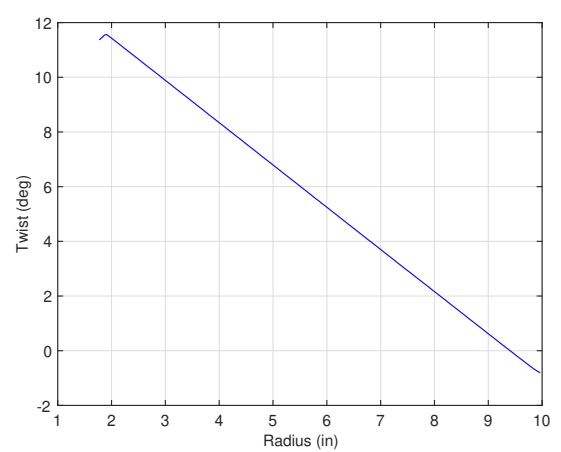

(b) Twist

Figure 15 KDE Rotor Geometry

\section{Acoustic Evaluation by CHARM / PSU-WOPWOP}

CHARM is a commercial rotorcraft aeromechanics software package marketed by Continuum Dynamics, Inc. (CDI). It is widely used in the rotorcraft industry. Under a NASA funded effort, CDI is developing new capabilities in CHARM for a new class of vehicles for urban air mobility. CHARM is a vortex-lattice solver with semi-empirical methods for aeromechanics prediction of rotorcraft. PSU-WOPWOP is an aeroacoustic code developed by Prof. Kenneth Brentner at PSU et al [13]. Unsteady blade loading predicted by CHARM is used by PSU-WOPWOP to predict rotor acoustics. Mr. Daniel Wachspress of CDI has conducted an acoustic evaluation of a subset of the KDE design candidates on a voluntary basis due to his technical interest. The acoustic evaluation by CHARM / PSU-WOPWOP could provide increased confidence in the CFD acoustic prediction by STAR-CCM+.

In this study, CHARM does not model the actual blade airfoil profiles, but instead models the airfoil profiles as a NACA 0012 airfoil with varying chord and twist as shown in Fig. 15. Because CHARM does not model the actual blade profiles, the absolute comparison with the CFD results is not considered. Rather, the trend in the CHARM results is sought for comparing with the CFD results.

Two vortex lattice models of the baseline KDE rotor are created with a one-row lattice and a five-row lattice to predict the propagation of the SPL at one diameter beneath the rotor. Figure 16 illustrates the two models in CHARM.

Figure 17]shows the overall and blade vortex interaction (BVI) SPLs of the baseline rotor computed with the one-row lattice model and five-row lattice model in the forward flight condition at $9.7 \mathrm{~m} / \mathrm{s}$, corresponding to an advance ratio of $\mu=0.1$ and a zero shaft angle or an attack of attack of $\alpha_{s}=0$. The overall SPLs for both models are very similar. Therefore, the one-row lattice model is sufficient for the acoustic evaluation. In the forward flight condition, the BVI SPL constitutes about $86 \%$ of the overall SPL of the baseline rotor.

Figure 18 shows the overall SPLs of the baseline and anti-phase rotor design candidates 2A, 3B, 4A, 4C, and 5A. The overall SPLs of the anti-phase rotors generally increase by $2 \mathrm{~dB}$ to $8 \mathrm{~dB}$. Compared to the CHARM result for design candidate 4A, which shows a $2 \mathrm{~dB}$ increase in the SPL, the same increase in the SPL is also seen in the CFD result as shown in Table 2. The general increase in the SPL could be due to the general increase in the thrust level produced by the anti-phase rotors. The CHARM results do not attempt to match the thrust level produced by the anti-phase rotors. The CHARM results also confirm the trend found in the CFD results that design candidate 4A generally produces better noise reduction than most of the other design candidates.

Another acoustic evaluation in CHARM is conducted at a forward flight speed of $16 \mathrm{~m} / \mathrm{s}$ corresponding to an advance ratio of $\mu=0.15$ and a shaft angle of $\alpha_{s}=6^{\circ}$. This flight condition is known to produce BVI in the HART rotor [11]. Figure 19] shows the overall SPLs of the anti-phase rotor design candidates. Both design candidates 2A and $3 \mathrm{~B}$ produce more noise than the baseline rotor by $2 \mathrm{~dB}$. On the other hand, the other design candidates produce less noise than the baseline rotor by as much as $4 \mathrm{~dB}$ with design candidate $4 \mathrm{~A}$. Both sets of CHARM results for two different forward flight conditions indicate that design candidate $4 \mathrm{~A}$ has lower noise characteristics than the other design candidates. This observation is consistent with the CFD, results which also indicate that the four-period TE waveform in general produces better noise reduction than the other TE waveforms. 

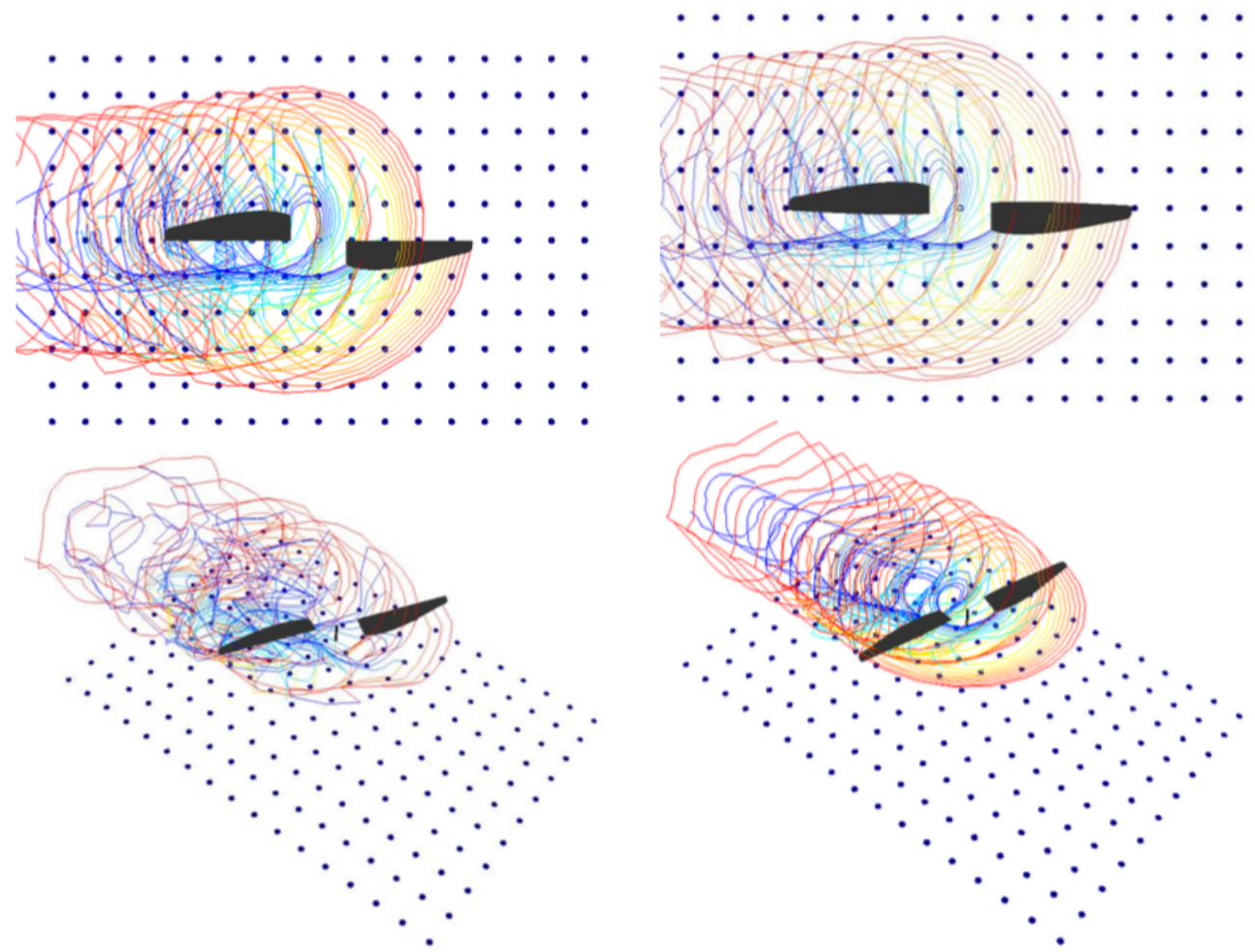

(a) One-Row Lattice Model

(b) Five-Row Lattice Model

Figure 16 CHARM Models of KDE Equivalent Baseline Rotors 


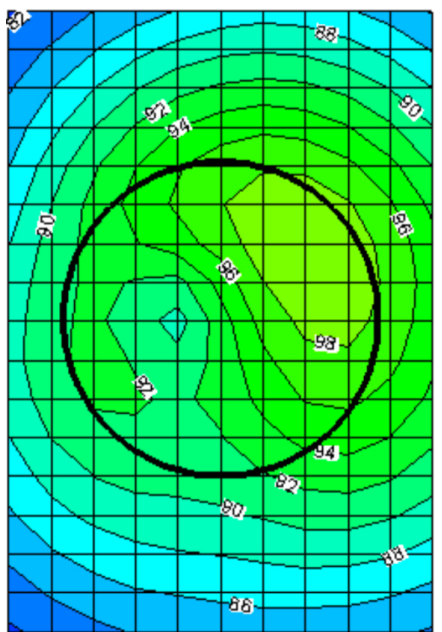

(a) Overall SPL One-Row Lattice Model

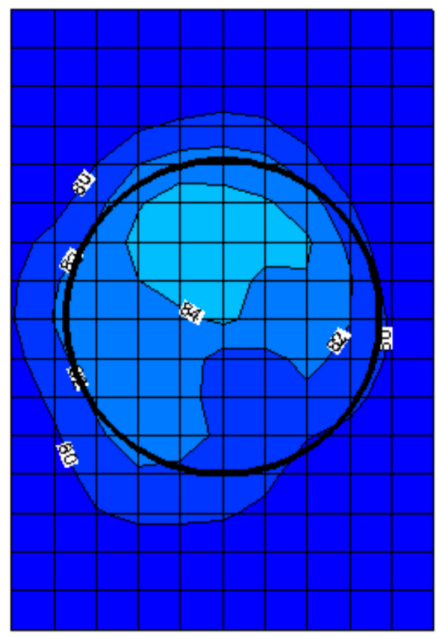

(c) BVI SPL One-Row Lattice Model
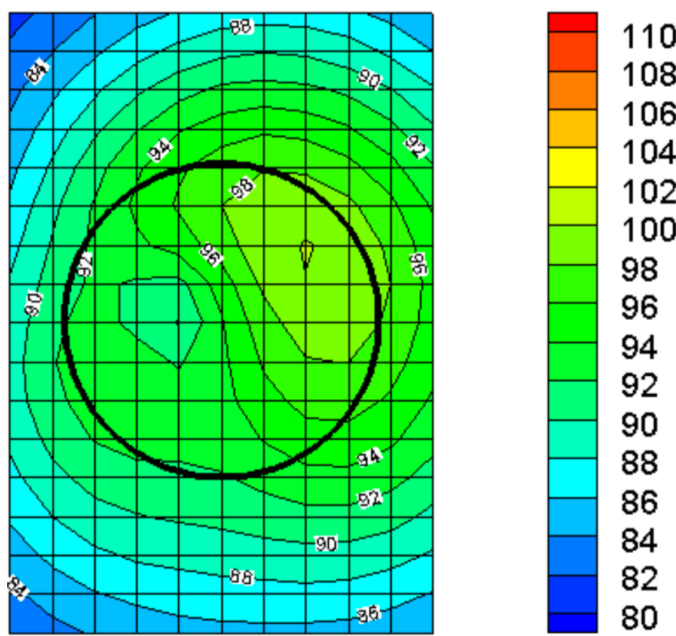

(b) Overall SPL Five-Row Lattice Model
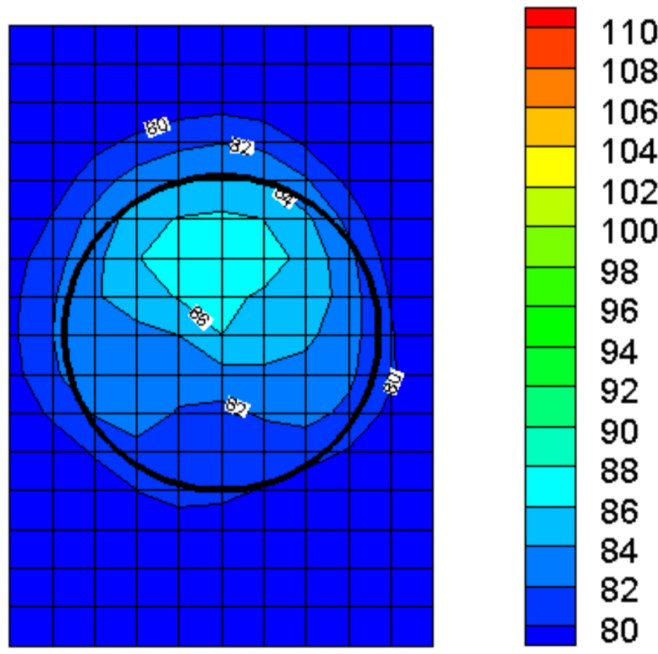

(d) BVI SPL Five-Row Lattice Model

Figure 17 SPL of Baseline Rotor at $9.7 \mathrm{~m} / \mathrm{s}$ and $\alpha_{s}=0^{\circ}$ Computed with One-Row and Five-Row Lattice Models 


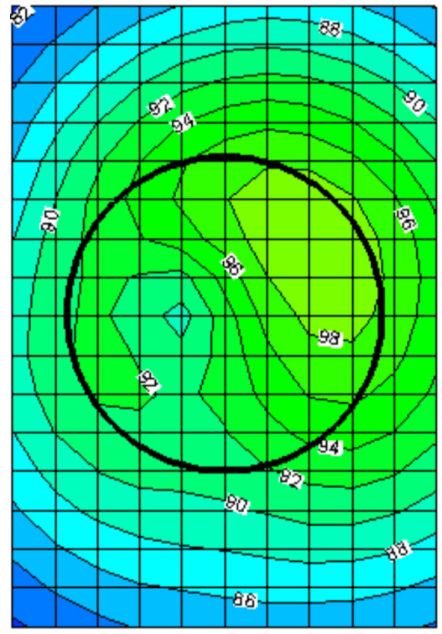

(a) Overall SPL Baseline

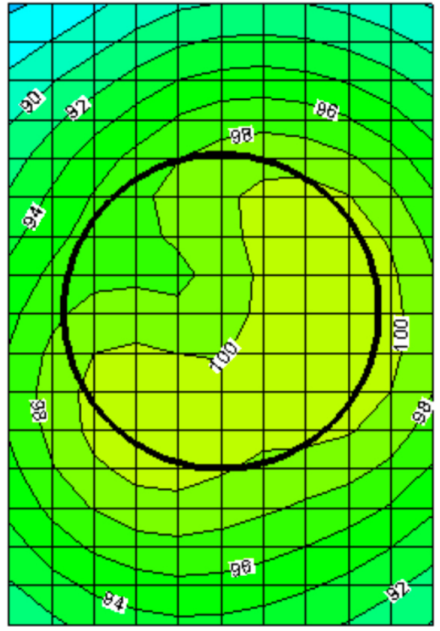

(c) Overall SPL 3B

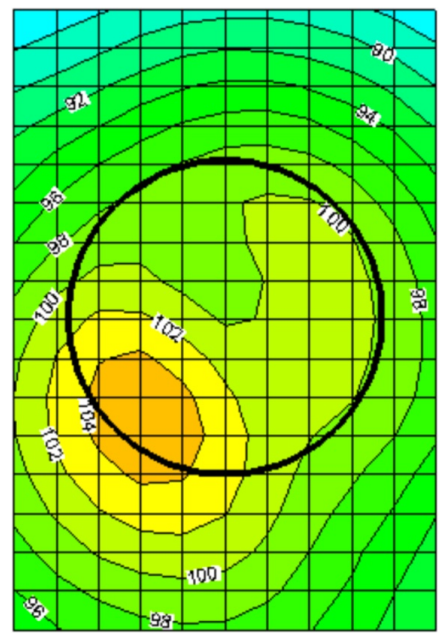

(e) Overall SPL 4C

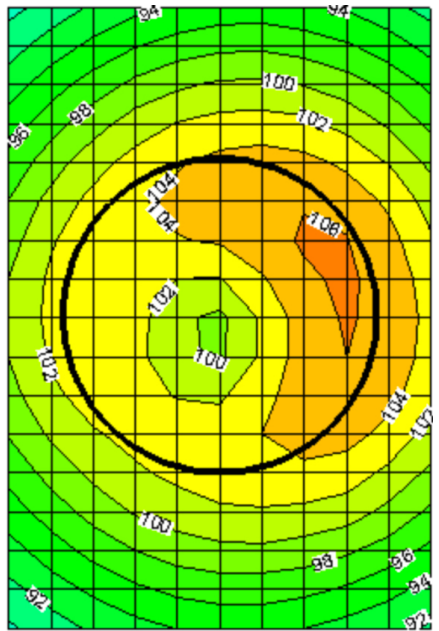

110

108

106

104

102

100

98

96

94

92

90

88

86

84

82

80

(b) Overall SPL 2A

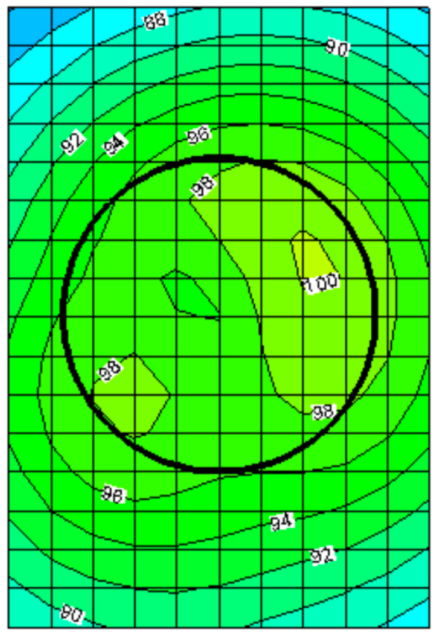

110

108

106

104

102

100

98

96

94

92

90

88

86

84

82

80

(d) Overall SPL 4A
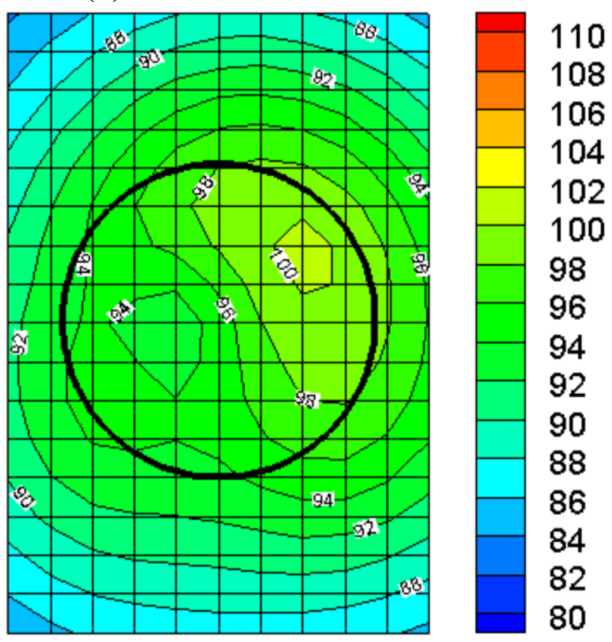

(f) Overall SPL 5A

Figure 18 SPL of Baseline and Anti-Phase Rotors at $9.7 \mathrm{~m} / \mathrm{s}$ and $\alpha_{s}=0^{\circ}$ 


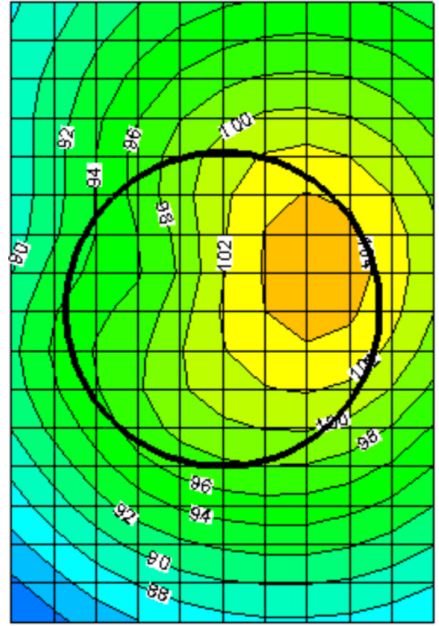

(a) Overall SPL Baseline

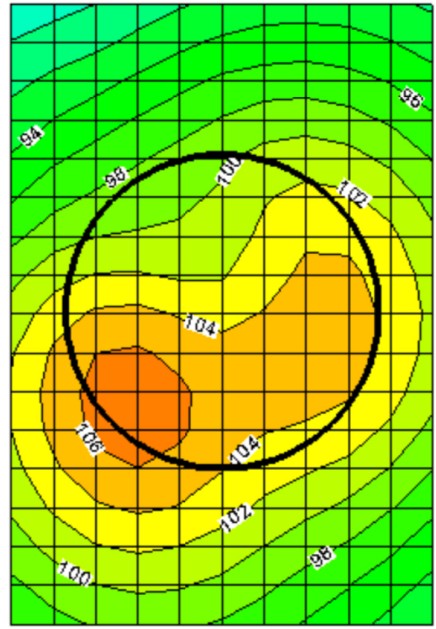

(c) Overall SPL 3B

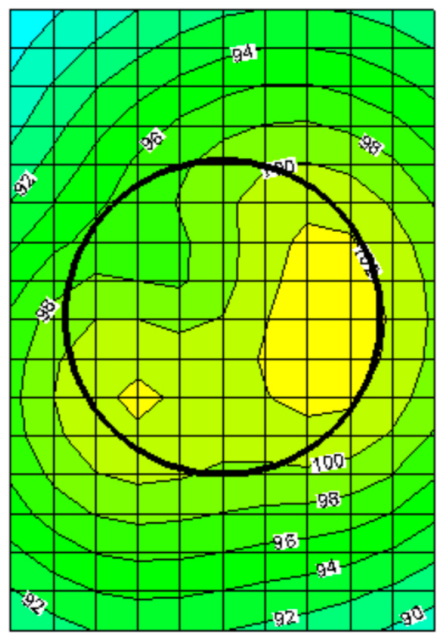

(e) Overall SPL 4C

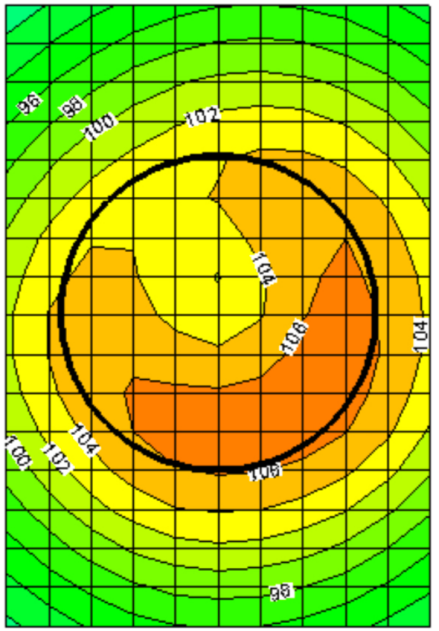

110

108

106

104

102

100

98

96

94

92

90

88

86

84

82

80

(b) Overall SPL 2A

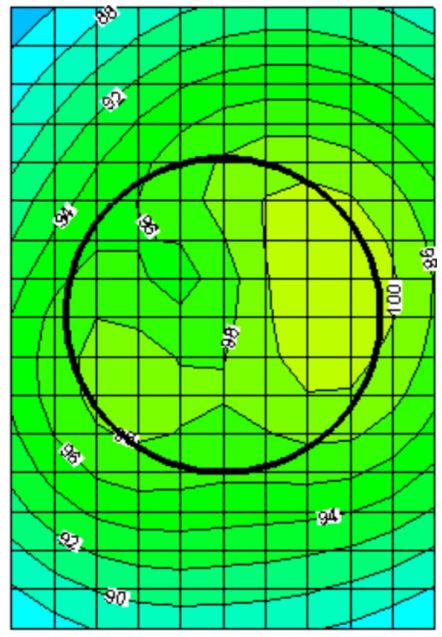

110

108

106

104

102

100

98

96

94

92

90

88

86

84

82

80

(d) Overall SPL 4A

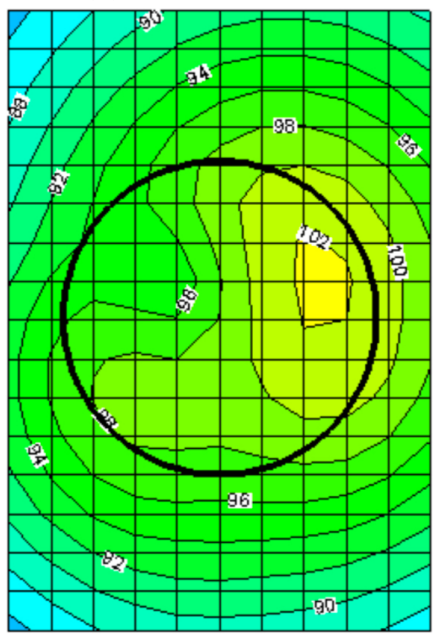

110

108

106

104

102

100

98

96

94

92

90

88

86

84

82

80

(f) Overall SPL 5A

Figure 19 SPL of Baseline and Anti-Phase Rotors at $16 \mathrm{~m} / \mathrm{s}$ and $\alpha_{s}=6^{\circ}$ 


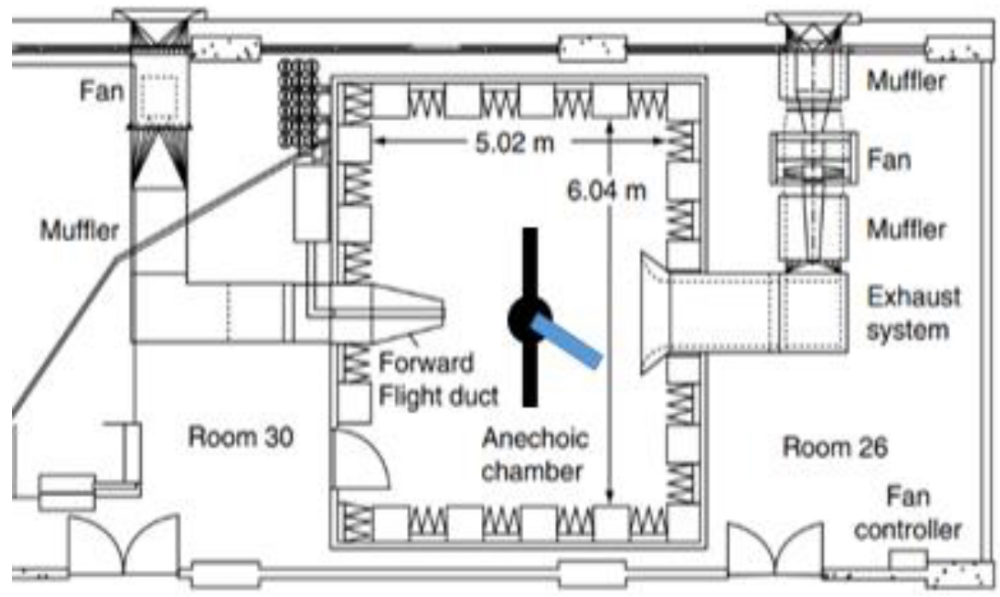

Figure 20 Anechoic Chamber with Forward Flight Duct at Pennsylvania State University

\section{Experimental Investigation}

\section{A. Experimental Setup}

An experimental investigation of the noise reduction characteristics of the anti-phase rotor is conducted in the PSU anechoic chamber called the PSU High Speed Jet Noise Facility. Figure 20 and Figure 21 how the anechoic chamber an the microphone array setup, respectively. The anechoic chamber has a theoretical cut-off frequency of $500 \mathrm{~Hz}$ and contains a forward flight duct that allows for different flight conditions to be tested such as forward flight and side gust conditions alongside hover.

PSU has developed a novel acoustic test rig that allows for the creation of an experimental acoustic sphere around a noise source. The test rig pictured in Fig. 22(a) consists of a half-circular microphone array, shown in Fig. 22.b), that has 19 housings for $1 / 2$ inch microphones. The separation angle between each microphone is $10^{\circ}$. The array is installed on a post and is able to rotate (manually) around the axis. Rotating the whole microphone array around the pole allows for the creation of a complete experimental acoustic sphere around the noise source. The test rig has the flexibility to test any noise source including UAV rotors in multiple configurations, such as: single rotor, co-axial counter-rotating (CCR), co-axial same direction (CSD), and scaled down helicopter rotors.

The rotor system available is of a co-axial configuration. Each rotor has two blades. The two rotors can be activated or shut down independently to allow for single or co-axial rotor configuration tests. The angular velocity is measured by two optical RPM sensors. Thrust and power are quantified by two sets of force and torque sensors, respectively. Details are seen in Fig. 23 Both a RPM and a thrust control are developed for the test setup. For the tests, only the bottom rotor is activated and used.

A background noise measurement has been conducted in preparation for the acoustic test. The background noise level of the test chamber is documented as the baseline acoustic reading. Figure 24 shows the background noise from microphones 12 to 14 when the motor spins at 4000 RPM without the rotor installed. The microphone choice displayed here is arbitrary. A Fast Fourier Transform (FFT) and a running average are used to generate Figure 24 The limitation of such a method could be that a periodic noise will be displayed to be around its average value. Two major components on top of the baseline reading are able to be seen. They are the DC power supply noise and the high frequency noise. The DC power supply sits in the anechoic chamber with the experimental setup due to current limitations of available connection points. This is set to be mitigated and improved in the future. The high frequency noise is determined to be the noise from the motors. This high frequency noise will be present throughout the series of tests. The removal of this high frequency noise from the experimental data plots in subsequent cases would be more representative of deltas produced by the setup. This method of displaying data is preferred by rotorcraft acousticians.

The tested blades for the anechoic chamber and data acquisition verification are modified to incorporate the anti-phase trailing edge designs. The first design of the anti-phase trailing edge rotor blades is based on the KDE rotor. However, this geometry used in the CFD simulations somehow is different from the actual KDE rotor that will be tested. So the acoustic measurement data is not presented in this paper.

The second design of the anti-phase trailing edge rotor blades will be based on the APC rotor design candidates $4 \mathrm{H}$, 


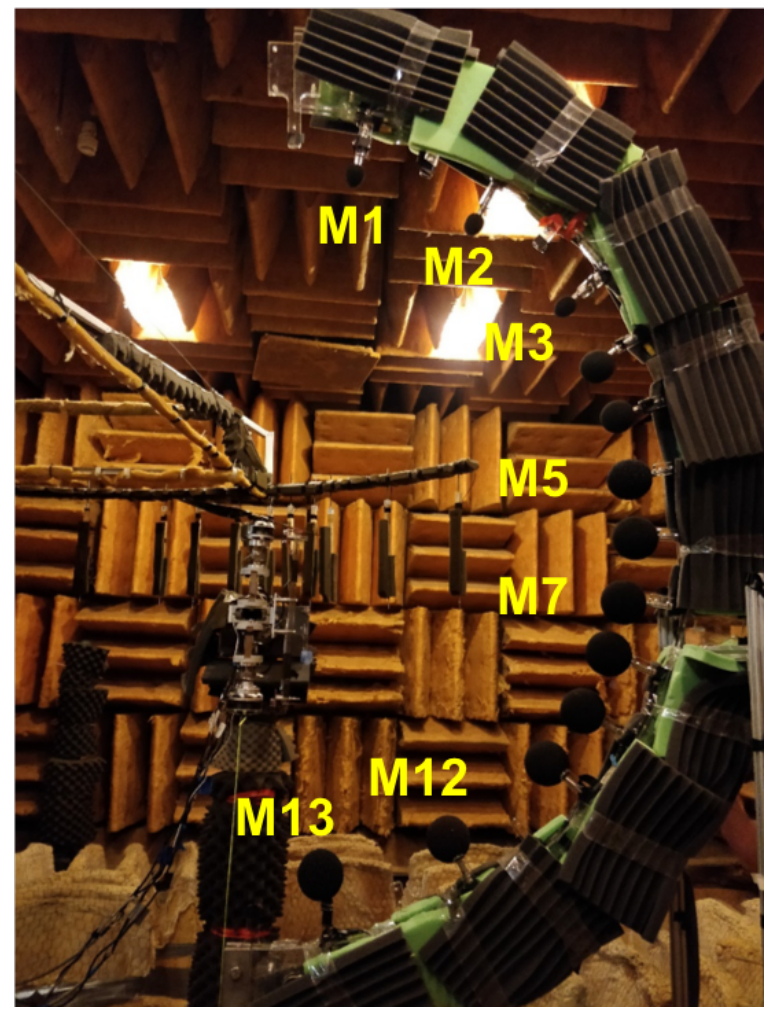

Figure 21 Anechoic Chamber

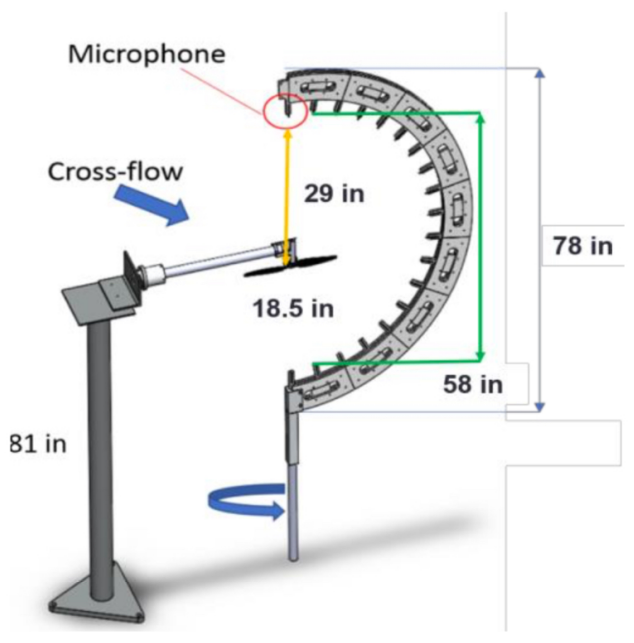

(a) Acoustic Test Rig

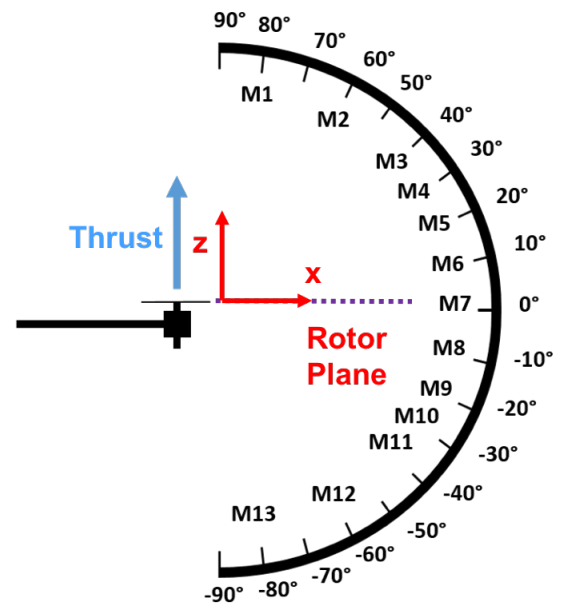

(b) Microphone Array

Figure 22 Acoustic Test Rig with Microphone Array 


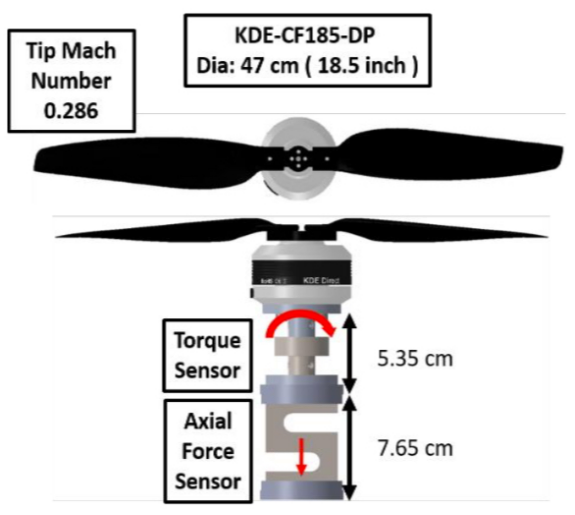

Figure 23 Acoustic Test Rotor and Sensors

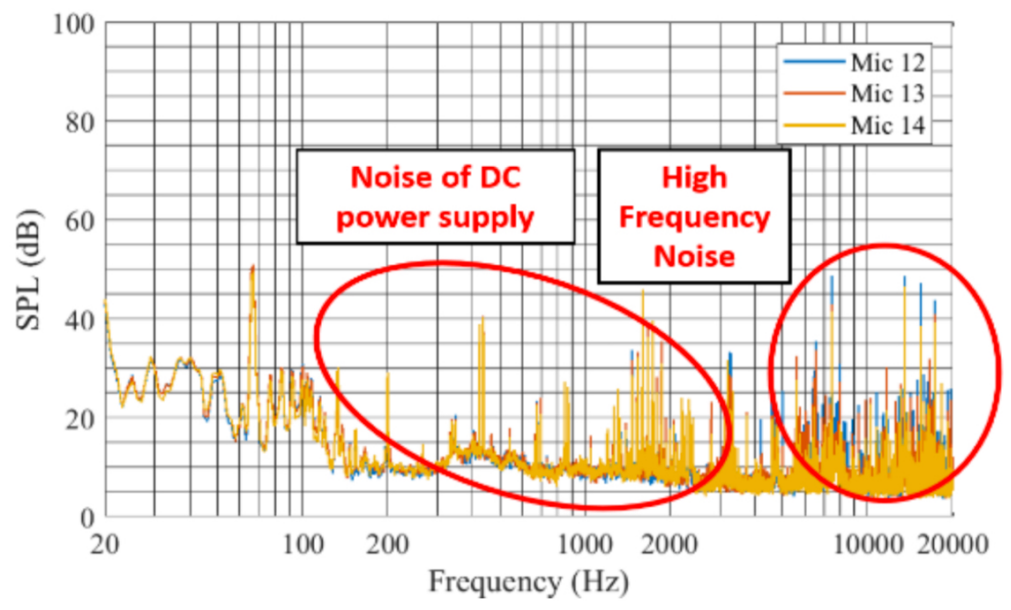

Figure 24 Background Noise Level Mic 12 - 14, No Blades, @ 4000 RPM and Wind Tunnel Speed of 0 m/s 


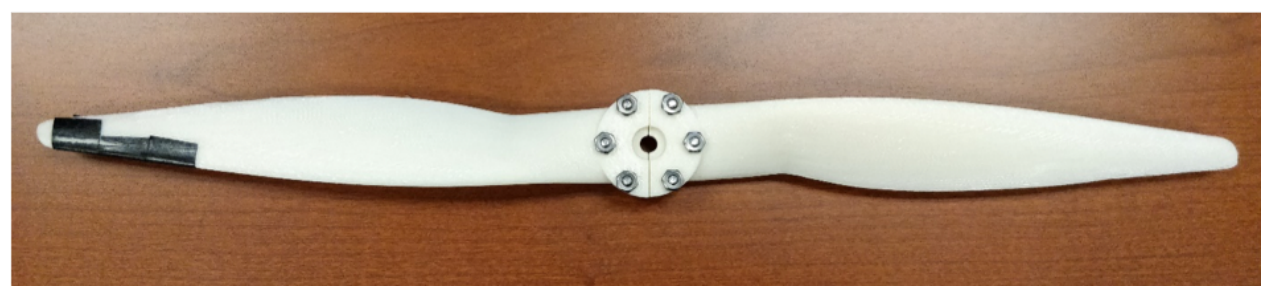

(a) APC Baseline Rotor Blade

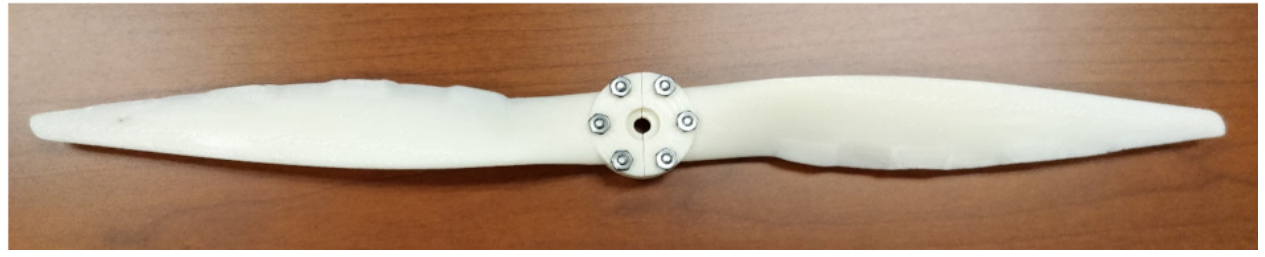

(b) APC Anti-Phase Rotor Design Candidate 4I

Figure 25 APC Baseline and Anti-Phase Rotor Design Candidate 4I

and 4I. The APC baseline, 4H and 4I geometries are manufactured by 3D printing, which are shown in Fig. 25. A few pieces of tape are attached at the tip of the APC baseline rotor blade for force balance.

\section{B. Experimental Results}

\section{Hover Condition}

APC baseline and anti-phase rotor design candidates $4 \mathrm{H}$ and $4 \mathrm{I}$ are tested at $3200 \mathrm{RPM}$. Figure 26 shows the Overall Sound Pressure Level (OASPL) directivity plot. The asymmetric blade is the design candidate $4 \mathrm{H}$. The symmetric blade is the design candidate 4I. The experimental data at hover condition show the anti-phase rotor design candidates of APC do not provide a better noise level. The experimental data of the APC anti-phase rotor in the hover condition does not agree with the CFD results. However, as noted by the PSU research staff, acoustic tests in the hover condition generally cannot provide conclusive experimental results, because it is well-known that the downwash created by the rotor causes the flow in the anechoic chamber to recirculate back to the rotor. This flow recirculation, which is not present in the actual flight operation, causes uncertainty in the experimental data.

\section{Forward Flight Condition at $9.7 \mathrm{~m} / \mathrm{s}$}

APC baseline and anti-phase rotor design candidates, $4 \mathrm{H}$ and 4I, are also tested at $3200 \mathrm{RPM}$ with forward flight condition $9.7 \mathrm{~m} / \mathrm{s}$. Figure 27 shows the OASPL directivity plot. The anti-phase rotor design candidate $4 \mathrm{H}$ offers a minor noise suppression across a wide range of azimuthal angles. Figure 28 shows the comparison of SPL at MIC4 between the anti-phase rotor design candidates, $4 \mathrm{H}$ and 4I, and APC baseline. It clearly shows a significant noise reduction by the anti-phase rotor design candidates, 4H and 4I, across frequency range from $2000 \mathrm{~Hz}$ to $4000 \mathrm{~Hz}$. The APC anti-phase $4 \mathrm{H}$ rotor produces as much as a $6 \mathrm{~dB}$ noise reduction. The experimental data confirms the noise suppression of the anti-phase rotor design concept. 


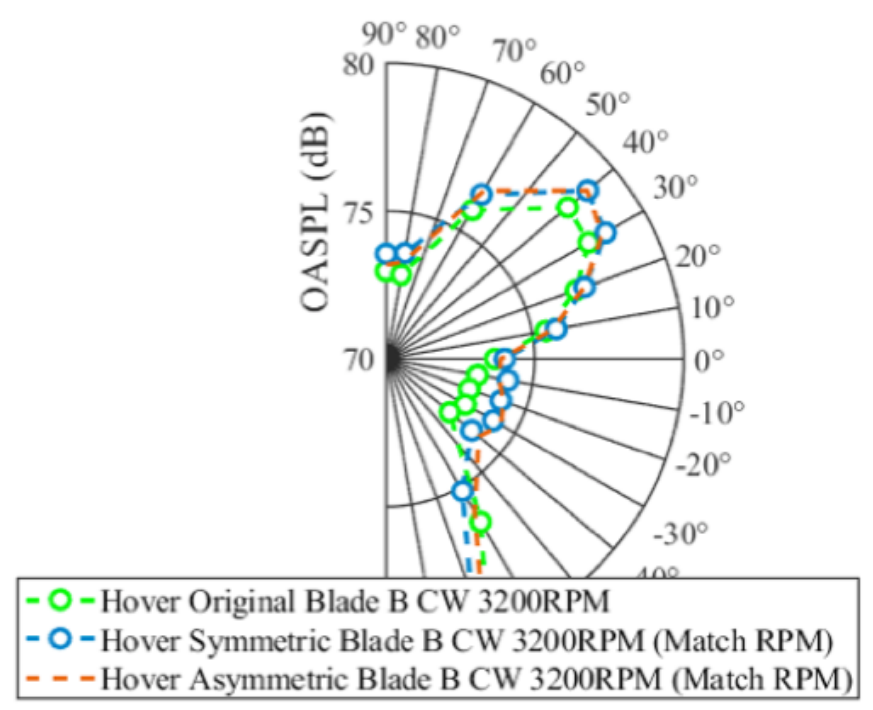

Figure 26 APC Baseline and Anti-Phase Rotor Design Candidates 4H and 4I OASPL Directivity Plot at Hover Condition

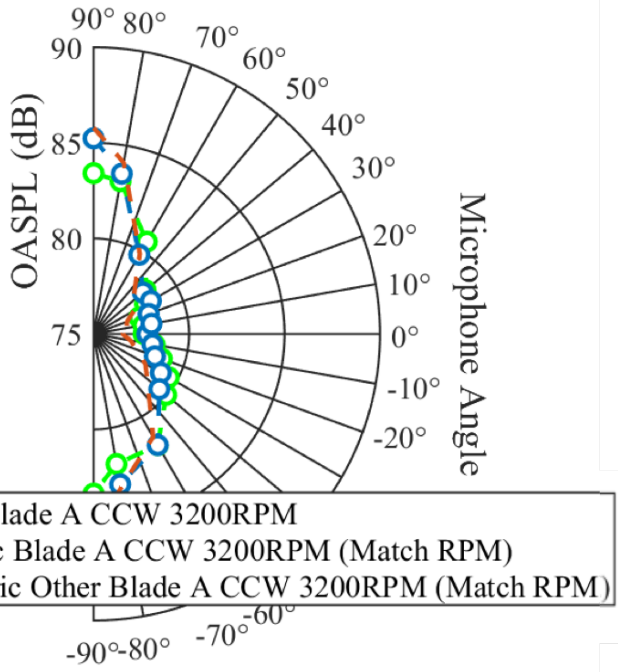

Figure 27 APC Baseline and Anti-Phase Rotor Design Candidates 4H and 4I OASPL Directivity Plot at Forward Flight Condition $9.7 \mathrm{~m} / \mathrm{s}$ 


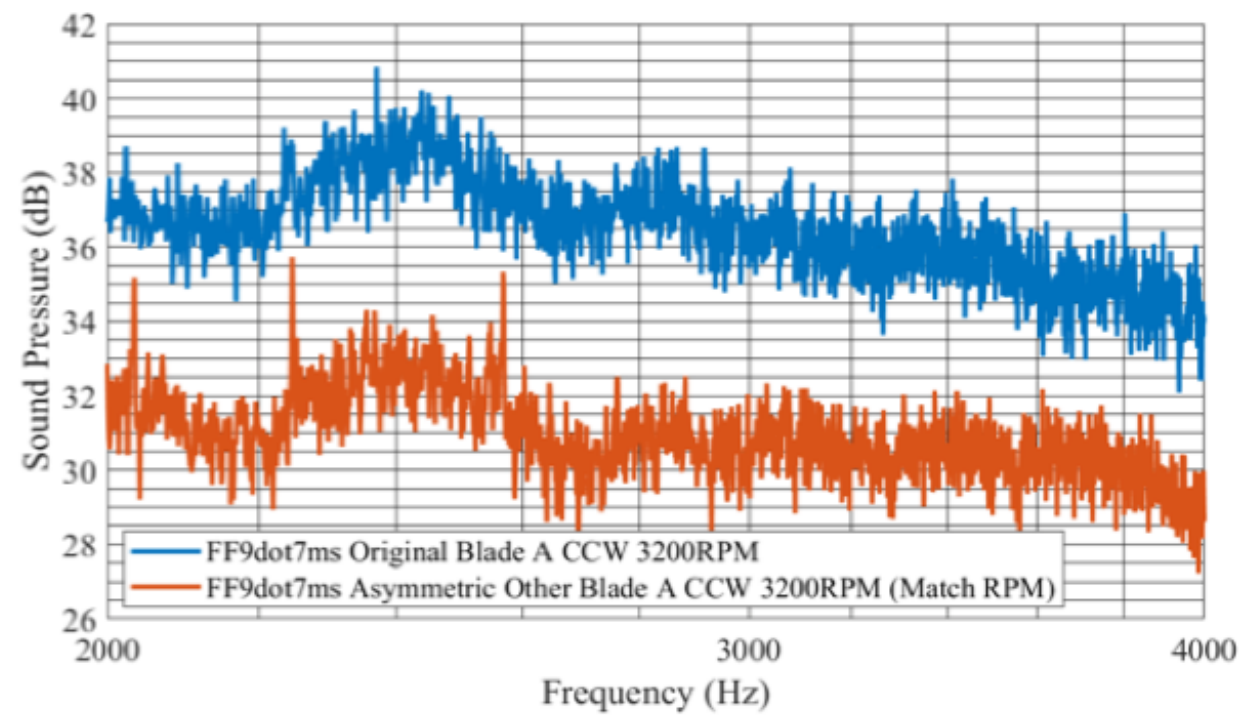

(a) $4 \mathrm{H}$

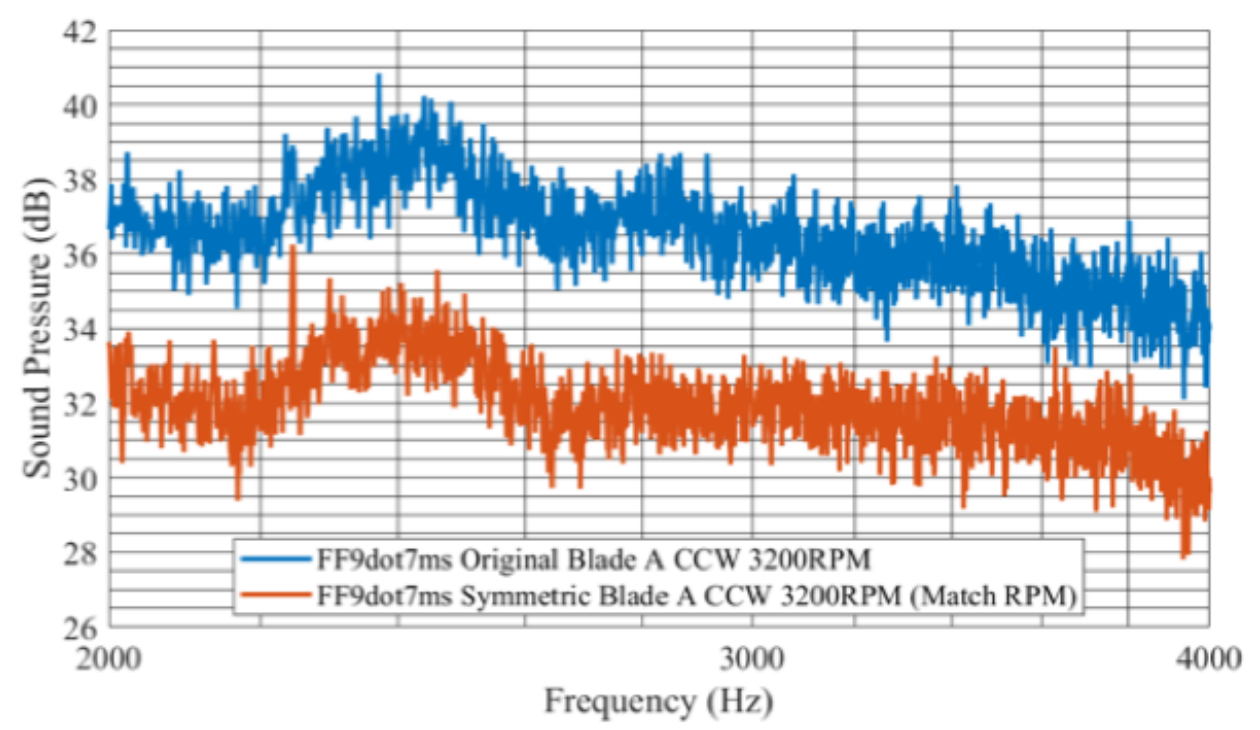

(b) $4 \mathrm{I}$

Figure 28 APC Baseline and Anti-Phase Rotor Design Candidates 4H and 4I SPL at MIC 4 at Forward Flight Condition $9.7 \mathrm{~m} / \mathrm{s}$ 


\section{Conclusions}

An acoustic optimization study is performed in this paper. A CFD investigation is conducted to seek to optimize the anti-phase alternating trailing edge patterns for rotor noise suppression. The design objective is to maximize the noise reduction perceived by the community while maintaining the aerodynamic thrust. The investigation is performed using a three-dimensional (3D) Unsteady Reynolds-Averaged Navier-Stokes (URANS) commercial solver STAR-CCM+ with $k-\omega$ Shear Stress Transport (SST) turbulence model and Ffowcs-Williams and Hawkings (FW-H) formula to obtain aerodynamic thrust and far-field noise level. An initial parametric acoustic study is conducted for 13 anti-phase design candidates of KDE rotor. The CAD geometry of the standard rotor is initially furnished by PSU and is obtained by laser scanning because the KDE rotor has a proprietary geometry. These design candidates include different alternating trailing edge (TE) waveforms, TE segment lengths, TE deflection amplitudes, and transition characteristics. The best design candidate for the KDE rotor has a four-period TE waveform which results in a reduction in far-field noise level of $2.1 \mathrm{~dB}$ in the hover condition and a reduction of $1.1 \mathrm{~dB}$ in the forward flight condition at $9.7 \mathrm{~m} / \mathrm{s}$. In addition, an rotor acoustic prediction is conducted using a commercial rotorcraft aeromechanics code called CHARM produced by Continuum Dynamics Inc. (CDI). CHARM has an acoustic predictive capability provided by PSU-WOPWOP, an acoustic code developed by PSU coupled to CHARM. CHARM is used to evaluate the acoustic characteristics of a subset of the KDE anti-phase rotor design candidates. The results computed by CHARM also confirm a similar noise reduction level and trend as the CFD results. A further parametric acoustic study is conducted for a different rotor manufactured by APC. Six APC rotor design candidates are simulated. The best design candidate, $4 \mathrm{H}$, for the APC rotor results in a reduction in far-field noise level of $4.0 \mathrm{~dB}$ in the hover condition and a reduction of $1.3 \mathrm{~dB}$ in the forward flight condition at $9.7 \mathrm{~m} / \mathrm{s}$.

A series of acoustic experiments in the PSU anechoic chamber have been conducted. At hover condition, the anti-phase rotor design candidates do not provide better noise levels. However, the data at hover condition are inconclusive due to the flow recirculation in the anechoic chamber. At a forward flight condition $9.7 \mathrm{~m} / \mathrm{s}$, the anti-phase rotor design candidate, $4 \mathrm{H}$, for the APC rotor can operate at a lower noise level than the APC baseline at the same RPM. The APC anti-phase $4 \mathrm{H}$ rotor produces as much as $6 \mathrm{~dB}$ noise reduction at a frequency range from $2000 \mathrm{~Hz}$ to $4000 \mathrm{~Hz}$.

\section{Acknowledgment}

The authors wish to acknowledge NASA Ames Center Innovation Fund to support of this work. The authors also acknowledge PSU Dr. Jose Palacios, Mr. Raja Akif Raja Zahirudin and Mr. Sihong Yan for providing the acoustic experiment data. The authors also acknowledge CDI for their CHARM simulation support.

\section{References}

[1] Moore, M. D., "Personal Air Vehicles: a Rural/Regional and Intra-Urban On-Demand Transportation System," Journal of AIAA, Vol. 2646, 2003.

[2] Duffy, M. J., Wakayama, S. R., and Hupp, R., "A Study in Reducing the Cost of Vertical Flight with Electric Propulsion,” 17th AIAA Aviation Technology, Integration, and Operations Conference, 2017, p. 3442.

[3] Antcliff, K. R., Moore, M. D., and Goodrich, K. H., "Silicon Valley as an Early Adopter for On-Demand Civil VTOL Operations,"16th AIAA Aviation Technology, Integration, and Operations Conference, 2016, p. 3466.

[4] “Volocopter VC2,” https://www.volocopter.com/assets/pdf/2017_04_Design_specifications_2X.pdf, 2017. Accessed: 2018-063.

[5] Wright, S., “The Acoustic Spectrum of Axial Flow Machines,” Journal of Sound and Vibration, Vol. 45, No. 2, 1976, pp. $165-223$.

[6] Kurtz, D., and Marte, J., “A Review of Aerodynamic Noise from Propellers, Rotors, and Lift Fans,” 1970.

[7] Yung, H. Y., "Rotor Blade-Vortex Interaction Noise,” Progress in Aerospace Sciences, Vol. 36, No. 2, 2000 , pp. 97-115.

[8] Hardin, J. C., and Lamkin, S. L., “Concepts for Reduction of Blade/Vortex Interaction Noise,” Journal of Aircraft, Vol. 24, No. 2, 1987, pp. 120-125.

[9] Chen, P. C., Baeder, J. D., Evans, R. A., and Niemczuk, J., "Blade-Vortex Interaction Noise Reduction with Active Twist Smart Rotor Technology," Smart Materials and Structures, Vol. 10, No. 1, 2001, p. 77. 
[10] Cramer, N., Nguyen, N., Hernandez, S., and Storms, B., "Investigation of Anti-Phase Asymmetric Quiet Rotor Technology", AIAA Aeroacoustics Conference, AIAA-2019-1070, Jan. 2019.

[11] Menter, F., “Two-Equation Eddy-Viscosity Turbulence Models for Engineering Applications,” AIAA Journal, Vol. 32, No. 8, 1994, pp. 1598-1605.

[12] Ffowcs Williams, J. and Hawkings, D., "Sound Generation by Turbulence and Surfaces in Arbitrary Motion," Philosophical Transactions of Royal Society of London, Series A, Mathematical and Physical Sciences (1934-1990), 264:1151, 1969, pp. 321-342.

[13] Brès, G.A., Brentner, K.S., Perez, G., and Jones, H.E., "Maneuvering Rotorcraft Noise Prediction," Journal of Sound and Vibration, Vol. 39, No.3-5, August 2003, pp. 719-738.

[14] Splettstoesser, W., Kube, R., Wagner, W., Seelhorst, U., Boutier, A., Micheli, F., Mercker, E., and Pengel, K., "Key Results from a Higher Harmonic Control Aeroacoustic Rotor Test (HART)," Journal of the American Helicopter Society, Vol. 42, No. 1, 1997, pp. 58-78. 